\title{
A PROTOSTELLAR JET EMANATING FROM A HYPERCOMPACT H II REGION
}

\author{
Andrés E. Guzmán ${ }^{1}$, Guido Garay ${ }^{1}$, Luis F. Rodríguez ${ }^{2}$, Yanett Contreras ${ }^{3,4}$, Catherine Dougados ${ }^{1}$, and \\ SYlviE CABRIT ${ }^{5}$ \\ ${ }^{1}$ Departamento de Astronomía, Universidad de Chile, Camino el Observatorio 1515, Las Condes, Santiago, Chile \\ ${ }^{2}$ Instituto de Radioastronomía y Astrofísica (UNAM), Morelia 58089, México \\ ${ }^{3}$ CSIRO Astronomy and Space Science, P.O. Box 76, Epping 1710 NSW, Australia \\ ${ }^{4}$ Leiden Observatory, Leiden University, P.O. Box 9513, NL-2300 RA Leiden, The Netherlands \\ ${ }^{5}$ LERMA, Observatoire de Paris, UMR 8112 du CNRS, ENS, UPMC, UCP, 61 Av. de l'Observatoire, F-75014 Paris, France \\ Received 2016 April 16; revised 2016 May 22; accepted 2016 May 23; published 2016 August 1
}

\begin{abstract}
We present radio continuum observations of the high-mass young stellar object (HMYSO) G345.4938+01.4677 obtained using the Australia Telescope Compact Array (ATCA) at 5, 9, 17, and 19 GHz. These observations provide definite evidence that the outer and inner pairs of radio lobes consist of shock-ionized material being excited by an underlying collimated and fast protostellar jet emanating from a hypercompact $\mathrm{H}$ II region. By comparing with images taken 6 years earlier at 5 and $9 \mathrm{GHz}$ using the same telescope, we assess the proper motions (PMs) of the radio sources. The outer west and east lobes exhibit PMs of $64 \pm 12$ and $48 \pm 13$ mas yr $^{-1}$, indicating velocities projected in the plane of the sky and receding from G345.4938+01.4677 of 520 and $390 \mathrm{~km} \mathrm{~s}^{-1}$, respectively. The internal radio lobes also display PM signals consistently receding from the HMYSO with magnitudes of $17 \pm 11$ and $35 \pm 10 \mathrm{mas} \mathrm{yr}^{-1}$ for the inner west and east lobes, respectively. The morphology of the outer west lobe is that of a detached bow shock. At 17 and $19 \mathrm{GHz}$, the outer east lobe displays an arcuate morphology also suggesting a bow shock. These results show that disk accretion and jet accelerationpossibly occurring in a very similar way compared with low-mass protostars-is taking place in G345.4938 +01.4677 despite the presence of ionizing radiation and the associated hypercompact $\mathrm{H}$ II region.
\end{abstract}

Key words: ISM: individual objects (G345.4938+01.4677) - ISM: jets and outflows - radio continuum: ISM stars: formation

\section{INTRODUCTION}

In spite of the preponderant influence of high-mass stars $\left(M_{\star}>8 M_{\odot}\right)$ on the evolution of galaxies, with them being the progenitors of core collapse supernovae and the main sources of chemical enrichment, turbulence, and mixing in the interstellar medium, the details of their formation are not well understood (Zinnecker \& Yorke 2007; Tan et al. 2014, p. 149). The formation of high-mass stars is more difficult to study than that of low-mass stars due to their relative scarcity, larger distances, shorter formation timescales $\left(\leqslant 10^{6}\right.$ years), and large extinction toward their birth places $\left(A_{V}>10\right)$.

The currently accepted evolutionary path of high-mass stars begins inside dense and massive molecular cores where highmass young stellar objects (HMYSOs) accrete at rates between $10^{-5}$ to $10^{-3} M_{\odot} \mathrm{yr}^{-1}$ (Tan et al. 2014, p. 149). The young stars finish their Kelvin-Helmholtz contraction very rapidly and reach the main sequence (Norberg \& Maeder 2000; Keto \& Wood 2006). At this point, the star radiates extreme ultraviolet (UV) photons that ionize its surroundings, producing a hypercompact (HC) $\mathrm{H}$ II region. $\mathrm{HC} \mathrm{H}$ II regions are observationally characterized by sizes $\lesssim 0.03 \mathrm{pc}$, densities $n_{e}>$ $10^{6} \mathrm{~cm}^{-3}$, emission measures $>10^{8} \mathrm{pc} \mathrm{cm}^{-6}$, and hydrogen recombination line (HRL) widths $\gtrsim 50 \mathrm{~km} \mathrm{~s}^{-1}$ (Kurtz 2000; Hoare et al. 2007, p. 181). Eventually, the accretion flow will stop and the UV radiation and winds from the recently formed star will disperse the rest of the envelope material, leaving the O-type star surrounded by a classical H II region (Zapata et al. 2010).

Recent observational evidence gathers in favor of accretion as the preferred high-mass star formation mechanism despite the radiation pressure, the thermal pressure of the heated protostellar material, and the ionized gas pressure. In combination, these processes could theoretically reverse the accretion flows (Larson \& Starrfield 1971; Wolfire \& Cassinelli 1987). High accretion rates have been invoked in high-mass star formation in order to overcome the radiation pressure and to choke the development of an $\mathrm{H}$ II region (Garay \& Lizano 1999). However, what appears to be crucial in the solution of the theoretical problems described above is the nonspherical character of accretion. Disk accretion, in particular, is an effective way to circumvent the radiation and ionized gas pressure, allowing the accreting material to reach the young high-mass stars much more easily by flowing inward, mainly through the plane perpendicular to the angular momentum vector of the system (Nakano 1989; Kuiper et al. 2011). Confirming this theoretical picture entails finding observational evidence of disk accretion toward HMYSOs. Currently, most of the evidence is indirect and consists of contracting motions of molecular clumps, poorly collimated molecular outflows, and rotationally flattened molecular structures (see Guzmán et al. 2014 and references therein).

For O-type stars, we expect that a fraction $\gtrsim 50 \%$ of the final stellar mass will be accreted after the Kelvin-Helmholtz contraction and after the star starts to produce ionizing radiation (Hosokawa \& Omukai 2009; Zhang et al. 2014). If material during this stage is accreted through a disk, then it is pertinent to look for evidence of disk accretion toward $\mathrm{HC} \mathrm{H}$ II regions and to settle the question as to whether or not accretion onto the HMYSO is maintained after stellar contraction and UV photon injection. Note that accretion through a disk may only choke the ionized region near the disk plane, allowing for $\mathrm{H}$ II region development in the polar regions (Keto 2007). HC H II regions indeed exhibit indirect evidence pointing in this direction, such as 
very high column densities $\left(N_{\mathrm{H}_{2}}>10^{23} \mathrm{~cm}^{-2}\right)$, high incidence $(>70 \%)$ of infalling motions of the surrounding molecular gas (Klaassen et al. 2012), molecular outflows, rotationally flattened molecular structures, and time-varying radio fluxes (GalvánMadrid et al. 2008).

Currently, the most direct way to assert that a high-mass star is accreting from a disk is by finding highly collimated jets (aperture $\leqslant 5^{\circ}$ ) with velocities comparable with the escape velocity of the central object (Livio 2009, pp. 3-9). While lowvelocity and poorly collimated outflows are produced by a variety of processes, like magnetic braking (Hennebelle et al. 2011), ionization feedback (Peters et al. 2012), and even mergers (Bally \& Zinnecker 2005), highly collimated and fast outflows seem to be more reliable signposts of disk accretion (Seifried et al. 2012). Observationally, collimated jets (e.g., Caratti o Garatti et al. 2015; Purser et al. 2016) and keplerianlike disks (e.g., Kraus et al. 2010; Sánchez-Monge et al. 2013; Johnston et al. 2015) have been observed to be associated with luminous HMYSOs, supporting the disk accretion scenario for high-mass stars.

In this work, we present new observations of the multiple radio source detected toward the HMYSO G345.4938 +01.4677 (G345.49+1.47 hereafter). ${ }^{6}$ With a bolometric luminosity of $\sim 32,000 L_{\odot}$ (assuming a distance of $1.7 \mathrm{kpc}$, López et al. 2011), G345.49+1.47 is the dominant central HMYSO of the $\sim 1000 M_{\odot}$ molecular clump associated with IRAS 16562-3959. The bolometric luminosity of the IRAS source is $(5-7) \times 10^{4} L_{\odot}$ (López et al. 2011). ${ }^{7}$ Five roughly aligned radio continuum sources were detected toward G345.49+1.47 by Guzmán et al. (2010). Four of these flank in an approximately symmetric manner a bright central radio source located at the position of the HMYSO. In addition, infrared (IR) 2MASS and GLIMPSE images show emission extending toward the east and along the direction of the string of radio sources. Guzmán et al. (2010) interpreted the emission from the central radio source as arising from an ionized protostellar jet and the rest of the sources as shock-ionized lobes. The IR appearance of G345.49+1.47 can be understood as the illuminated inner walls of an outflow cavity which contains the jet. This emission is enhanced toward the eastern side of the jet which corresponds-as inferred from observations of an associated molecular bipolar outflow (Guzmán et al. 2011) — to the blueshifted side of the jet. Similar interpretations have been proposed for other well-know HMYSOs associated with radio jets such as G343.1262 -00.0620 (also IRAS 16547-4247, Garay et al. 2003; Rodríguez et al. 2008).

In contrast to these interpretations, HRL observations of G345.49+1.47 (Guzmán et al. 2014) demonstrated that the central source is not a protostellar ionized jet but a photoionized $\mathrm{HC} \mathrm{H}$ II region. The question arises as to whether or not the radio lobes are being excited by an underlying (and still undetected) jet, which would be part of a purported disk-jet system within this $\mathrm{HC} \mathrm{H}$ II region. Evidence in this direction will support the adequacy of the disk-jet star formation mechanism in the high-mass case, even after the onset of ionizing radiation. Section 2 of this work describes the new

\footnotetext{
6 We use the nomenclature of the rms survey (http://www.ast.leeds.ac.uk/ rms, Lumsden et al. 2013) based on the Midcourse Space Experiment coordinates.

7 Note that the rms survey adopts a distance of $2.4 \mathrm{kpc}$ and a luminosity of $1.5 \times 10^{5} L_{\odot}$ for this HMYSO.
}

observations performed toward G345.49+1.47. Section 3 presents the results, and Section 4 discusses the interpretation and jet characteristics. We summarize our conclusions in Section 5 .

\section{OBSERVATIONS}

We observed G345.49+1.47 using the Australia Telescope Compact Array ${ }^{8}$ (ATCA) in October of 2014 and in March and May of 2015 under the project ID number C3006. We observed 3 runs of $12 \mathrm{hr}, 1$ using the $1.5 \mathrm{~km}$ configuration and the other 2 using the $6.0 \mathrm{~km}$ configuration. The phase center of the array was toward R.A. $=16^{\mathrm{h}} 59^{\mathrm{m}} 41^{\mathrm{s}} .61, \quad$ decl. $=-40^{\circ} 03^{\prime} 43^{\prime \prime} 4$ (J2000). We evenly split the time among two frequency settings using the Compact Array Broadband Backend (Wilson et al. 2011) without zooms. The first frequency setting simultaneously covers the $4.0-6.0 \mathrm{GHz}$ and $8.0-10.0 \mathrm{GHz}$ spectral windows using 2048 channels of $1 \mathrm{MHz}$ width. The second frequency setting simultaneously covers the $16.0-18.0 \mathrm{GHz}$ and $18.0-20.0 \mathrm{GHz}$ spectral windows, also using 2048 channels of $1 \mathrm{MHz}$ width. Hereafter, we refer to each of these four frequency intervals as 5, 9, 17, and $19 \mathrm{GHz}$, respectively.

We calibrated the data using the Miriad software (Sault et al. 1995). The flux scale was determined by comparing with the calibrator PKS 1934-638. We corrected for atmospheric and antenna based gain variations by observing the gain calibrator PKS 1729-37 regularly every 8 minutes. The fluxes measured toward PKS 1729-37 were 1.19, 1.27, 1.31, and $1.29 \mathrm{Jy}$ at $5,9,17$, and $19 \mathrm{GHz}$, respectively, which are within the observed flux variations. ${ }^{9}$ Deconvolution and imaging was performed using the Common Astronomy Software Applications (CASA, McMullin et al. 2007).

We also re-reduced archival data ${ }^{10}$ taken between 2008 October and 2009 February toward G345.49+1.47 and originally published by Guzmán et al. (2010). The source PKS 1740-517 was used as a gain calibrator. Since the radio emission toward G345.49+1.47 is dominated by a central, compact source of $\sim 13 \mathrm{mJy}$ at $9 \mathrm{GHz}$ with a spectral index of $\sim 1$ (see Section 3), we were able to apply phase selfcalibration. This new reduction and imaging of the $9 \mathrm{GHz}$ data reaches a noise level similar to that presented in Guzmán et al. (2010) but with less artifacts. In particular, we do not recover the three aligned sources mentioned by Guzmán et al. (2010) located 4 " north of their outer east lobe. Therefore, and also because these sources were aligned along one of the ridges of the dirty beam pattern, we conclude that they are likely spurious.

Table 1 gives the synthesized beams and the noise (measured as the rms deviations in areas devoid of sources) of the images taken at 5, 9, 17, and $19 \mathrm{GHz}$. The first and second rows of Table 1 list the parameters of the data taken between 2008-2009 and 2014-2015, respectively. We refer hereafter to these two epochs as 2008/09 and 2014/15. Note that no data were taken at 17 and $19 \mathrm{GHz}$ in the 2008/09 epoch. The sensitivity difference between the two epochs is due primarily to the backend bandwidth, being $128 \mathrm{MHz}$ in the 2008/09

\footnotetext{
8 The Australia Telescope Compact Array is part of the Australia Telescope National Facility which is funded by the Commonwealth of Australia for operation as a National Facility managed by CSIRO.

9 http://www.narrabri.atnf.csiro.au/calibrators/

10 This paper includes archived data obtained through the Australia Telescope Online Archive (http://atoa.atnf.csiro.au).
} 
Table 1

Observational Parameters

\begin{tabular}{|c|c|c|c|c|c|c|c|c|}
\hline \multirow{3}{*}{ Epoch } & \multicolumn{4}{|c|}{ Synthesized Beam (BMAJ × BMIN, P.A.) } & \multicolumn{4}{|c|}{ Noise } \\
\hline & \multicolumn{4}{|c|}{$\left(" \prime \times{ }^{\prime \prime},{ }^{\circ}\right)$} & \multicolumn{4}{|c|}{$\left(\mu \mathrm{Jy} \mathrm{beam}^{-1}\right)$} \\
\hline & $5 \mathrm{GHz}$ & $9 \mathrm{GHz}$ & $17 \mathrm{GHz}$ & $19 \mathrm{GHz}$ & $5 \mathrm{GHz}$ & $9 \mathrm{GHz}$ & $17 \mathrm{GHz}$ & $19 \mathrm{GHz}$ \\
\hline $2008 / 09$ & $2.74 \times 1.74,2$ & $1.59 \times 1.05,3$ & $\cdots$ & $\cdots$ & 91 & 75 & $\cdots$ & $\cdots$ \\
\hline $2014 / 15$ & $2.49 \times 1.36,3$ & $1.59 \times 0.89,3$ & $0.83 \times 0.44,6$ & $0.78 \times 0.39,-3$ & 18 & 17 & 30 & 27 \\
\hline
\end{tabular}

observations versus $\sim 2 \mathrm{GHz}$ in the $2014 / 15$ epoch. Fully calibrated and reduced images are available through the Dataverse. $^{11}$

\section{RESULTS}

The upper panel of Figure 1 shows a contour map of the 17 and $19 \mathrm{GHz}$ data combined taken during the 2014/15 epoch with labels for each of the detected sources. Clearly detected are the five sources reported by Guzmán et al. (2010), named outer east, inner east, central, inner west, and outer west and marked as OE, IE, C, IW, and OW, respectively. Roughly aligned with these sources and $\sim 5^{\prime \prime}$ east of OW, we detect an additional knot denoted $\mathrm{W}_{1}$. In addition, we detect three other sources in the field numbered from 1 to 3 in decreasing declination order. These sources were below the $4 \sigma$ level in the $9 \mathrm{GHz}$ images of Guzmán et al. (2010), and therefore they were not reported. The $\mathrm{W}_{1}$ knot is detected in the $2008 / 099 \mathrm{GHz}$ image at $\sim 5 \sigma$ but, due to the lower fidelity of the images presented in Guzmán et al. (2010), it was not reported as a separate source.

Figure 1 also shows (middle panel) a three-color near-IR image of $\mathrm{G} 345.49+1.47$ made using $\mathrm{J}_{-}, \mathrm{H}-$, and $\mathrm{K}_{S^{-}}$band observations obtained from the Vista Variables in the ViaLactea survey ${ }^{12}$ (VVV, Minniti et al. 2010) public data. The three-color image shows the scattered light from the inner walls of the outflow cavity associated with the blueshifted side of the bipolar outflow (Guzmán et al. 2011). The three-color image also shows a near-IR source detected in $\mathrm{K}_{S}$ band whose position (R.A. $=16^{\mathrm{h}} 59^{\mathrm{m}} 41^{\mathrm{s}} .645$, decl. $=-40^{\mathrm{d}} 03^{\mathrm{m}} 43$ ! $^{\prime} 64$ ) is coincident with the central radio source $\mathrm{C}$ and has an estimated apparent magnitude of $K_{S} \approx 12.4$. This near-IR source-better displayed in panels (c) and (d) - is likely the near-IR counterpart of the embedded HMYSO G345.49+1.47, also associated with the mid-IR source G345.4938+01.4677-Src\#2 detected by Mottram et al. (2007). Note that the nearest 2MASS source (16594202-4003450) is located toward the bright tip of the outflow cavity rather than toward source $C$.

\subsection{Flux Density and Morphology of the Radio Emission}

The five radio sources detected toward G345.49+1.47 during the 2008/09 epoch are detected unambiguously in the $2014 / 15$ images. We were also able to detect at a level of $7 \sigma$ or better four additional sources due to the improved sensitivity of the 2014/15 observations: those marked in Figure 1 with numbers 1,2 , and 3 , and knot $\mathrm{W}_{1}$, situated at 17 !' 1 from source $\mathrm{C}$ in the P.A. $=-87^{\circ}$ direction. Knot $\mathrm{W}_{1}$ is located between sources IW and OW and is approximately aligned with them.

\footnotetext{
11 http://dx.doi.org/10.7910/DVN/EMZPWU

12 http://vvvsurvey.drupalgardens.com/
}

Tables 2 and 3 list the positions and primary beam corrected flux densities, ${ }^{13}$ respectively, of all the detected sources. We used two-dimensional (2D) Gaussian fittings to calculate the positions and the uncertainties of sources $1,2,3, \mathrm{~W}_{1}$, and $\mathrm{C}$, and of the inner lobes using the imfit task within CASA. The uncertainty in the position of a Gaussian source is given approximately by (Condon 1997)

$$
\eta \sigma_{\mathrm{G}}\left(\sqrt{N_{\mathrm{eff}}} \mathrm{SNR}_{\mathrm{p}}\right)^{-1}
$$

where $\sigma_{\mathrm{G}}$ is the position standard deviation of the Gaussian $\left(\sigma_{\mathrm{G}}=\theta_{\mathrm{FWHM}} / 2 \sqrt{2 \ln 2}\right), N_{\mathrm{eff}}$ is the quotient between the source and beam solid angles, $\mathrm{SNR}_{\mathrm{p}}$ is the peak intensity of the source divided by the rms deviation of the image, and $1<\eta<2 \sqrt{2}$ is a correction factor that accounts for noise correlation on scales comparable with the beam size. For unresolved sources, $\eta \sim 1$.

The positions listed in Table 2 for the outer lobes (OE and $\mathrm{OW}$ ) correspond to the peak intensity determined using the tool maxfit in CASA. To estimate the uncertainty in these positions, we explore two approaches. In the first approach, we note that although the $\mathrm{OE}$ and $\mathrm{OW}$ lobe images have welldefined intensity peaks, their morphologies are not adequately represented by single Gaussians. This is because these sources are spatially resolved by our observations. Therefore, we use two Gaussians to model each lobe: one of them fits the emission near the intensity peak while the other reproduces the more extended and resolved emission from the lobe. For example, the skewed appearance of the OW lobe (suggesting a bow shock) is better reproduced by an extended Gaussian $(\approx 2$ !" 5 FWHM) plus a compact source $(\approx 0$ !' 8 FWHM) shifted west with respect to the center of the more extended Gaussian by $\sim 1^{\prime \prime}$. We approximate the uncertainty of the position of the peak as the uncertainty of the position of the compact Gaussian. This procedure takes into account the fact that a non-negligible fraction of the peak comes from a diffuse component, whereas direct application of Equation (1) without correction would underestimate the peak position uncertainty. The second approach consists of using the clean components and the synthesized beam to generate a model image for each lobe. To this model image we add simulated random noise (correlated within the synthesized beam) at the level indicated in Table 1. Then, we use maxfit to calculate the position of the peak for each of these simulated images. The standard deviation of these positions is our uncertainty estimation of the peak position of each lobe.

We find that the uncertainties derived using the first method are larger than those determined using the second method by about a factor of 2 . We decide to follow a more conservative

\footnotetext{
13 ATCA primary beam responses obtained from http://www.narrabri.atnf. csiro.au/observing/users_guide.
} 

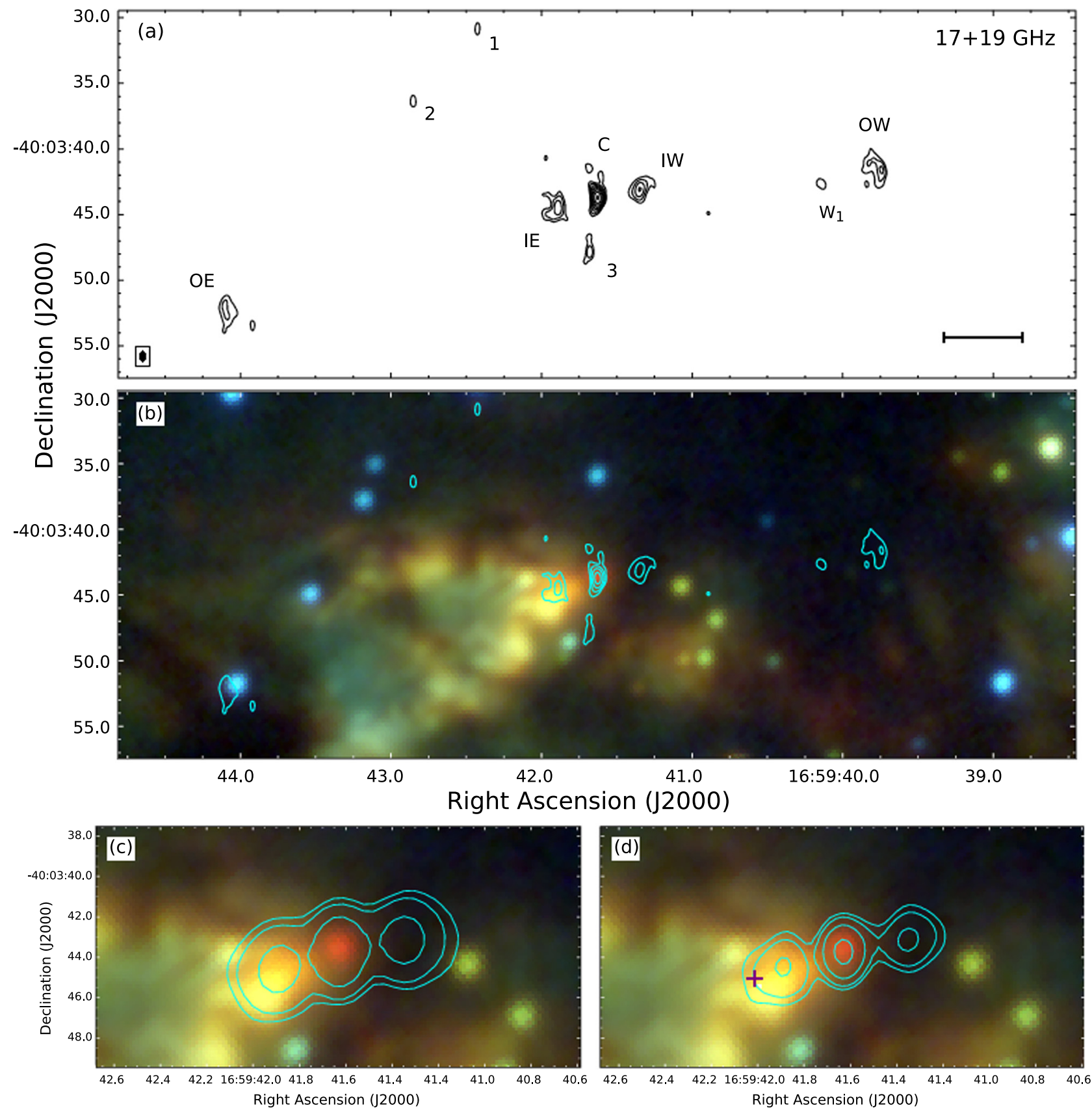

Figure 1. Panel (a) Contour map of the radio continuum emission observed at 17 and $19 \mathrm{GHz}$ combined. Contour levels: $7 \sigma \times 2^{i}$ for $i=1-8$ and $\sigma=20 \mu \mathrm{Jy}$. Labels are attached to each of the sources (Tables 2 and 3). The bar located in the bottom right corner of the map measures $0.05 \mathrm{pc}$ at $1.7 \mathrm{kpc}$. Panel (b) Three-color image of G345.49+1.47 made using J (blue), $\mathrm{H}$ (green), and $\mathrm{K}_{S}$ (red) data from the VVV survey. Cyan contours are as in panel (a) but drawn at every second level starting from the first. Panels (c) and (d) show a zoom-in into the region adjacent to G345.49+1.47 with superimposed cyan contours of the 5 and $9 \mathrm{GHz}$ data, respectively. Contour levels for panels (c) and (d) are given by $\left(3+4^{i}\right) \times 50 \mu \mathrm{Jy}$ with $i=0-2$ and $i=0 \ldots 3$, respectively. The purple cross in panel (d) indicates the position of $2 \mathrm{MASS}$ $16594202-4003450$.

approach and use the first method for the position uncertainty of the outer lobes. These uncertainties are given in Table 2 .

The flux densities listed in Table 3 are derived from Gaussian fittings for sources C, IE, and IW at 5 and $9 \mathrm{GHz}$. At 17 and $19 \mathrm{GHz}$ the inner lobes IE and IW show a compact component, with a deconvolved size of $\sim 0$ "! 8, within significant extended diffuse emission that cannot be adequately modeled as a Gaussian. Therefore, we calculate their fluxes integrating over $5^{\prime \prime} \times 5^{\prime \prime}$ boxes. We also calculate the flux densities of the outer lobes at all frequencies by integrating the intensity within boxes of $9^{\prime \prime} \times 9^{\prime \prime}$. Note that the flux density of the OW lobe includes the emission from $\mathrm{W}_{1}$. Source $\mathrm{W}_{1}$ appears to be unresolved and blended with the rest of the OW lobe emission at $9 \mathrm{GHz}$. Therefore, in Table 3, we report only its peak intensity. We also note that source $\mathrm{W}_{1}$ is detected in the $9 \mathrm{GHz} 2008 / 09$ images just below the $5 \sigma$ level, but it was not deemed significant and therefore not reported in Guzmán et al. (2010).

Figure 2 shows the inner region of G345.49+1.47 where sources C, IE, and IW are clearly distinguishable at the four 
Table 2

Position of the Radio Sources

\begin{tabular}{|c|c|c|c|c|c|}
\hline \multirow{3}{*}{ Source } & \multirow{3}{*}{ Epoch } & \multicolumn{4}{|c|}{ R.A. and decl. (J2000) } \\
\hline & & \multicolumn{4}{|c|}{$16: 59: \ldots,-40: 03: \ldots$} \\
\hline & & $5 \mathrm{GHz}$ & $9 \mathrm{GHz}$ & $17 \mathrm{GHz}$ & $19 \mathrm{GHz}$ \\
\hline \multirow[t]{2}{*}{$\mathrm{C}$} & $2008 / 09$ & $41^{\mathrm{s}} 623 \pm 0.001,433^{\prime \prime} 65 \pm 0 . \prime 02$ & $41.6305 \pm 0.0004,43{ }^{\prime \prime} 638 \pm 0$ ". 007 & $\cdots$ & $\cdots$ \\
\hline & $2014 / 15$ & $41.6273 \pm 0.0005,43.73 \pm 0.02$ & $41.6290 \pm 0.0002,43.728 \pm 0.006$ & $41.62760 \pm 0.00005,43.732 \pm 0.002$ & $41.62755 \pm 0.00004,43.705 \pm 0.001$ \\
\hline \multirow[t]{2}{*}{ IE } & $2008 / 09$ & $41.880 \pm 0.003,44.33 \pm 0.04$ & $41.885 \pm 0.005,44.33 \pm 0.04$ & $\cdots$ & $\cdots$ \\
\hline & $2014 / 15$ & $41.894 \pm 0.003,44.61 \pm 0.04$ & $41.896 \pm 0.003,44.58 \pm 0.04$ & $41.897 \pm 0.002,44.53 \pm 0.05$ & $41.894 \pm 0.002,44.51 \pm 0.05$ \\
\hline \multirow[t]{2}{*}{ IW } & $2008 / 09$ & $41.350 \pm 0.004,43.09 \pm 0.04$ & $41.359 \pm 0.005,43.01 \pm 0.03$ & $\ldots$ & $\ldots$ \\
\hline & $2014 / 15$ & $41.347 \pm 0.003,43.15 \pm 0.03$ & $41.349 \pm 0.003,43.12 \pm 0.03$ & $41.346 \pm 0.002,43.10 \pm 0.03$ & $41.350 \pm 0.002,43.07 \pm 0.04$ \\
\hline \multirow[t]{2}{*}{$\mathrm{OE}$} & $2008 / 09$ & $44.081 \pm 0.004,52.2 \pm 0.2$ & $44.081 \pm 0.005,52.0 \pm 0.1$ & $\ldots$ & $\ldots$ \\
\hline & $2014 / 15$ & $44.087 \pm 0.002,52.39 \pm 0.06$ & $44.099 \pm 0.001,52.23 \pm 0.05$ & $44.087 \pm 0.003,52.36 \pm 0.08$ & $44.091 \pm 0.004,52.39 \pm 0.07$ \\
\hline \multirow[t]{2}{*}{ OW } & $2008 / 09$ & $39.829 \pm 0.004,41.62 \pm 0.06$ & $39.830 \pm 0.007,41.4 \pm 0.1$ & $\ldots$ & $\ldots$ \\
\hline & $2014 / 15$ & $39.802 \pm 0.002,41.59 \pm 0.06$ & $39.764 \pm 0.001,41.44 \pm 0.04$ & $39.750 \pm 0.001,41.61 \pm 0.03$ & $39.753 \pm 0.001,41.62 \pm 0.05$ \\
\hline \multirow[t]{2}{*}{$\mathrm{W}_{1}$} & $2008 / 09$ & $\ldots$ & $40.18 \pm 0.0242 .5 \pm 0.4$ & $\ldots$ & $\cdots$ \\
\hline & $2014 / 15$ & $40.1207 \pm 0.007,42.71 \pm 0.2$ & $40.145 \pm 0.002,42.50 \pm 0.09$ & $40.139 \pm 0.001,42.75 \pm 0.05$ & $40.156 \pm 0.001,42.52 \pm 0.06$ \\
\hline \multicolumn{6}{|c|}{ Other sources } \\
\hline 1 & $2014 / 15$ & $\ldots$ & $42.4297 \pm 0.002,30.9 \pm 0.2$ & $42.427 \pm 0.002,30.74 \pm 0.06$ & $42.4238 \pm 0.0004,30.98 \pm 0.05$ \\
\hline 2 & $2014 / 15$ & $\ldots$ & (2. & $42.850 \pm 0.001,36.43 \pm 0.07$ & $42.855 \pm 0.001,36.27 \pm 0.05$ \\
\hline 3 & $2014 / 15$ & $\ldots$ & $41.710 \pm 0.002,48.05 \pm 0.05$ & $41.678 \pm 0.002,47.55 \pm 0.09$ & $41.6832 \pm 0.0005,47.95 \pm 0.02$ \\
\hline
\end{tabular}

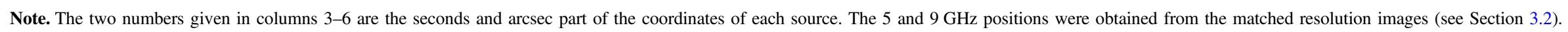


Table 3

Flux Densities

\begin{tabular}{lcccr}
\hline \hline Source & $\begin{array}{c}5 \mathrm{GHz} \\
(\mathrm{mJy})\end{array}$ & $\begin{array}{c}9 \mathrm{GHz} \\
(\mathrm{mJy})\end{array}$ & $\begin{array}{c}17 \mathrm{GHz} \\
(\mathrm{mJy})\end{array}$ & $\begin{array}{c}19 \mathrm{GHz} \\
(\mathrm{mJy})\end{array}$ \\
\hline Central & $7.6 \pm 0.2$ & $13.4 \pm 0.2$ & $20.8 \pm 0.1$ & $23.0 \pm 0.1$ \\
Inner east & $6.0 \pm 0.3$ & $6.5 \pm 0.4$ & $4.5 \pm 0.3$ & $4.2 \pm 0.3$ \\
Inner west & $6.0 \pm 0.3$ & $6.0 \pm 0.3$ & $4.4 \pm 0.2$ & $3.8 \pm 0.3$ \\
Outer east & $8.6 \pm 0.1$ & $8.6 \pm 0.1$ & $4.6 \pm 0.1$ & $5.1 \pm 0.1$ \\
Outer west & $7.4 \pm 0.1$ & $6.7 \pm 0.1$ & $4.7 \pm 0.2$ & $4.1 \pm 0.1$ \\
W $_{1}{ }^{\mathrm{a}}$ & $0.78 \pm 0.02$ & $0.61 \pm 0.02$ & $0.25 \pm 0.03$ & $0.20 \pm 0.03$ \\
Other sources & & & & \\
1 & $\ldots$ & $0.14 \pm 0.02$ & $0.38 \pm 0.06$ & $0.23 \pm 0.04$ \\
2 & $\ldots$ & $\ldots$ & $0.42 \pm 0.05$ & $0.44 \pm 0.06$ \\
3 & $\ldots$ & $0.26 \pm 0.03$ & $1.2 \pm 0.1$ & $0.95 \pm 0.06$ \\
\hline
\end{tabular}

Note.

a Intensity at peak.

observed frequencies. Source 3 is not detected at $5 \mathrm{GHz}$. The deconvolved angular size of source $\mathrm{C}$, calculated as the geometric mean of the deconvolved major and minor FWHM axes, are $390 \pm 80,195 \pm 10$, and $170 \pm 10$ mas at 9,17 , and $19 \mathrm{GHz}$, respectively, while it is unresolved at $5 \mathrm{GHz}$. While these angular sizes are typically smaller than half of the synthesized beam, source $\mathrm{C}$ is detected with a signal-to-noise ratio $\geqslant 20$ at all frequencies, which helps to reduce the uncertainty of the angular size estimations (see Condon et al. 1998, Section 5.2.4). These angular sizes are well fitted (residuals $<5$ mas) by a power law in frequency with spectral index $-1.1_{-0.4}^{+0.5}$. Figure 3 shows the spectral energy distribution and angular size versus frequency of source $\mathrm{C}$.

\subsection{Proper Motions (PMs)}

Sources C, IE, IW, OE, and OW are detected in our observations and were detected in the 2008/09 epoch at both 5 and $9 \mathrm{GHz}$, allowing us to study possible changes in their positions. Since the resolutions of the 2014/15 and 2008/09 images are slightly different, we convolve the images in order to match their synthesized beams through the procedure described in detail in the Appendix. The matched synthesized beam of the 5 and $9 \mathrm{GHz}$ images is 2 " $74 \times 1$ !" 74 with $\mathrm{P}$. A. $=2.9$ and $1^{\prime \prime} .68 \times 1{ }^{\prime \prime} 18$ with P.A. $=3.3$, respectively. The pixel sizes of the 5 and $9 \mathrm{GHz}$ images (the same for both epochs) are $0 . \prime 5$ and $0 . " 3$, respectively. These are $\sim 1 / 4$ of the smallest FWHM of the synthesized beams. Table 4 lists the displacements measured for each source detected at both epochs according to the difference between the coordinates given in Table 2.

Central Source. Source C exhibits the smallest displacement, of the order of 100 mas in the south direction at $9 \mathrm{GHz}$ and in the south-east direction at $5 \mathrm{GHz}$. This is somewhat expected due to the nature of source $\mathrm{C}$ : being a $\mathrm{HCH}$ II region we anticipate possible flux density variations (De Pree et al. 2014) but a relatively stable position. Note that the displacement of source $\mathrm{C}$ is approximately one tenth of the beam size and is roughly aligned with the $\mathrm{N}-\mathrm{S}$ maximum elongation direction of the synthesized beams. Therefore, it is possible that source C's apparent PM signal is an artifact of the observations and not related with a real displacement. For example, it could be due to using different gain calibrators in the two epochs of observation. Systematic astrometry shifts between different epochs of the order of one tenth of the size of the synthesized
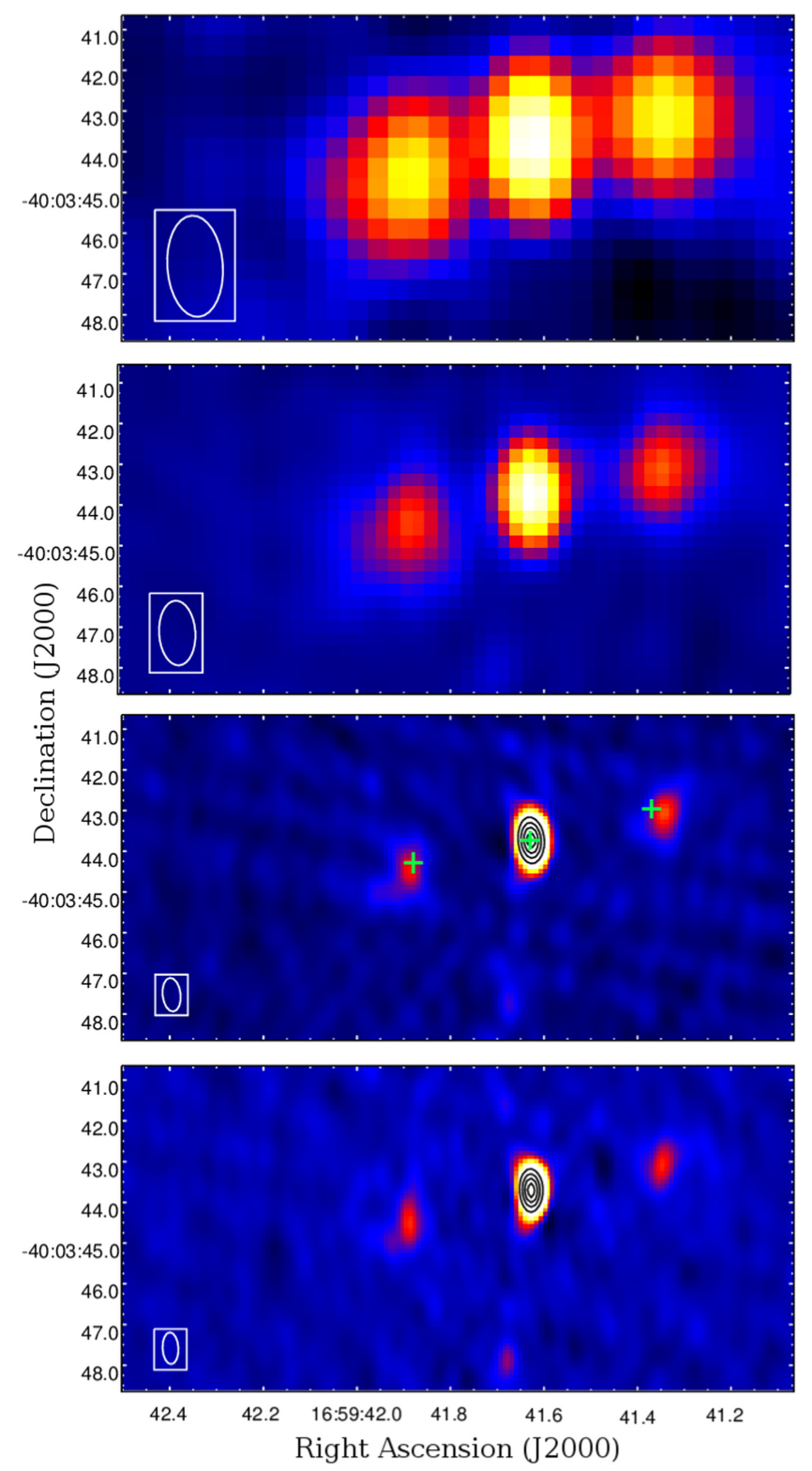

Figure 2. Color images of the radio continuum emission detected toward the central source and the two inner lobes. From top to bottom: 5, 9, 17, and $19 \mathrm{GHz}$. Black contours in the images at 17 and $19 \mathrm{GHz}$ are drawn at $30 \%$, $50 \%, 70 \%$, and $90 \%$ of the peak. Green crosses in the $17 \mathrm{GHz}$ image show the 2011 positions of sources IE, C, and IW determined by Purser et al. (2016).

beam are not rare. For instance, in a recent PM study presented by Masqué et al. (2015) on HH 80-81 using the VLA, they find shifts of approximately this magnitude as determined from comparing the positions of extragalactic radio sources. Unfortunately, there are no strong extragalactic sources in the field of G345.49+1.47 which could help us to align both epochs and correct for a global shift. If the PM of source $\mathrm{C}$ is real, then it would imply a speed of $\sim 100 \mathrm{~km} \mathrm{~s}^{-1}$ projected in the plane of the sky, which is unlikely. However, even in this case, ejecta from the HMYSO would presumably move at a velocity equal to the source's velocity plus the ejection velocity. Therefore, to determine the PMs of the lobes with respect to the central source, the PM of source $\mathrm{C}$ should be subtracted from the PMs of the rest of the sources, whether or 

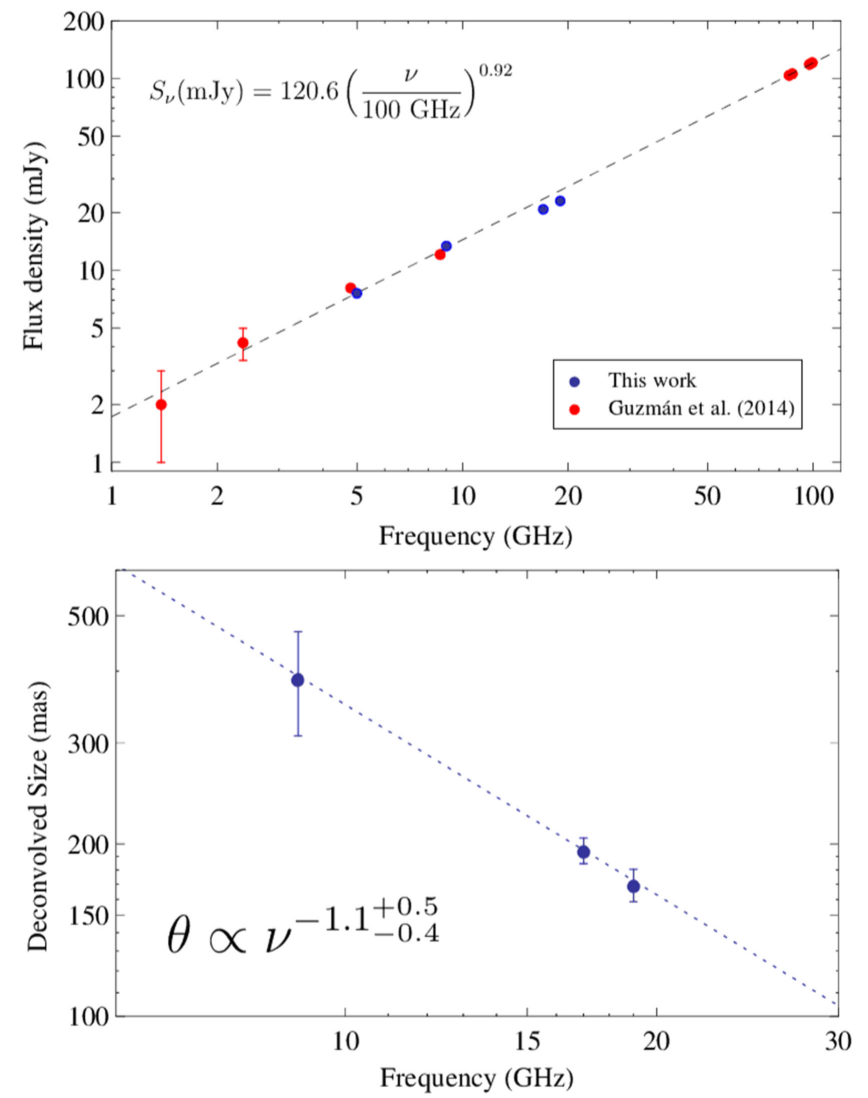

Figure 3. Top panel: spectrum of source C, including all previous data. The dashed line shows the spectrum derived in Guzmán et al. (2014). Bottom panel: angular size spectrum of source $\mathrm{C}$. The dotted line is the best power-law fit to the geometric mean of the deconvolved major and minor FWHM axes.

not the former is due to a systematic astrometry error. Hereafter, we subtract source C's displacement from that of the rest of the sources.

Inner Lobes. Figure 4 compares the 5 and $9 \mathrm{GHz}$ images taken in both epochs toward the inner sources (C, IE, and IW). The arrows in the lower panels represent the PM signals of the inner lobes and of source $\mathrm{C}$, given in Table 4. The ellipses show the $1 \sigma$ uncertainty regions. We find that the IE lobe has a PM equivalent to a velocity in the plane of the sky of $\sim 300 \mathrm{~km} \mathrm{~s}^{-1}$, with a more clear detection than that of the IW lobe. The PM signal of the IW lobe is weaker, being close to $2 \sigma$ at $9 \mathrm{GHz}$ and only slightly above $1 \sigma$ at $5 \mathrm{GHz}$. Figure 2(c) also shows the peak position of the inner lobes determined from observations at $17 \mathrm{GHz}$ carried out during 2011 (Purser et al. 2016). The clear displacement observed between our data and those taken independently provides further support for the PM of the inner lobes.

Outer Lobes. The morphology of the external lobes OE and OW is considerably more complex than that of the inner lobes. Images and contour maps of the $9 \mathrm{GHz}$ emission from the $\mathrm{OW}$ and $\mathrm{OE}$ lobes in the two epochs are shown, respectively, in Figures 5 and 6. We emphasize three characteristics of the OW lobe'(s) emission: (i) the shape of the main part of the lobe resembles a detached or bow-shaped shock, (ii) the peak of the emission has displaced away from the central source by $\sim 1^{\prime \prime}$, and (iii) the presence of the secondary knot $\mathrm{W}_{1}$ (see Figure 5). As shown in Figure 6, the OE lobe's shape is better defined in the 2014/15 images respect to the 2008/09 images. While the displacement of the peak position of the OW lobe is readily patent, that of the OE lobe is less clear. We note that the OE lobe is likely interacting with a dense gas structure which appears as a dark patch in the IR three-color image of Figure 1. Furthermore, the shape of the $\mathrm{OE}$ emission at $9 \mathrm{GHz}$ seems to form an arc curving away from source $\mathrm{C}$. The dashed magenta line in the top left panel of Figure 6 shows schematically the location of this arc. A possible interpretation is that it corresponds to a detached shock produced by the interaction of jet material with a stationary and dense cloudlet, as in the model of Schwartz (1978).

The rich structure of the $\mathrm{OE}$ and $\mathrm{OW}$ lobes introduces an additional difficulty when measuring displacements between two epochs. Therefore, we use two methods to measure their PMs: (i) by calculating the displacement of the peak of the emission, whose results are given in Table 4, and (ii) by maximizing the cross-correlation with respect to displacements between the two images. The cross-correlation method has been applied frequently in PM studies of extended emission associated with low-mass protostellar jets (e.g., Reipurth et al. 1996; Raga et al. 2012). The cross-correlation between two images $I_{1}$ and $I_{2}$ in a region $\mathcal{R}$ is defined as

$$
\rho_{12}(d x, d y)=\frac{\sum_{(x, y) \in \mathcal{R}} I_{1}(x, y) I_{2}(x-d x, y-d y)}{\left(\sum_{\mathcal{R}} I_{1}^{2}(x, y) \times \sum_{\mathcal{R}} I_{2}^{2}(x-d x, y-d y)\right)^{1 / 2}},
$$

where the sums run over all pixels with coordinates $(x, y)$ in $\mathcal{R}$ and $(d x, d y)$ is the displacement of image $I_{2}$ with respect to $I_{1}$. Clearly, we always have $\left|\rho_{12}\right| \leqslant 1$. If there is no noise and $\rho_{12}(\tilde{d x}, \tilde{d y})=1$, then the morphology of the emission inside $\mathcal{R}$ is preserved exactly between $I_{1}$ and $I_{2}$, and $I_{1}(x, y)=$ constant $\times I_{2}(x-\tilde{d} x, y-\tilde{d y})$. Therefore, in addition to the best-fit displacement, the maximum value attained by $\rho_{12}$ is a measure of the degree of similarity between the two epochs. We implement the cross-correlation and its maximization using $\mathrm{PDL}^{14}$ and the package Minuit. To evaluate the crosscorrelation at sub-pixel size accuracy, we linearly interpolate the images using the task interpND within PDL.

Table 5 gives the displacements calculated by maximization of the cross-correlation toward the OE and OW lobes. Columns 2 and 3 give the R.A. and decl. displacements for the $5 \mathrm{GHz}$ images and column 4 lists the value of $\boldsymbol{\rho}_{\max }$. Columns 5-7 give these same quantities but for the $9 \mathrm{GHz}$ images. To determine the uncertainty of each PM signal, we construct synthetic images of the $\mathrm{OE}$ and $\mathrm{OW}$ lobes convolving the clean components of these regions (obtained from the CASA task clean) and adding simulated Gaussian noise at the level given by Table 1 (correlated spatially according to the synthesized beams). We introduce artificial PM signals to each simulation by shifting one of the synthetic images by a random vector. We recover the PM signal through cross-correlation maximization and calculate its difference with the one introduced artificially. This difference $(\Delta \alpha, \Delta \delta)$ gives us an estimation of the uncertainty of the cross-correlation method. Repeating this process $N$ times generates a $N \times 2$ (rows $\times$ columns) matrix of differences, which we denote $\boldsymbol{D}$. The correlation matrix $\boldsymbol{D}^{\mathrm{T}} \boldsymbol{D}$ define the uncertainty ellipses of the cross-correlation method. For simplicity, in Table 5, we only give the projections of the

\footnotetext{
${ }^{14}$ http://pdl.perl.org/
} 
Table 4

Displacements of the Peak Position ${ }^{\mathrm{a}}$

\begin{tabular}{|c|c|c|c|c|}
\hline \multirow{2}{*}{ Source } & \multicolumn{2}{|c|}{$5 \mathrm{GHz}$} & \multicolumn{2}{|c|}{$9 \mathrm{GHz}$} \\
\hline & $\underset{\left({ }^{\prime \prime}\right)}{\Delta \text { R.A. }}$ & $\underset{\left({ }^{\prime \prime}\right)}{\Delta \text { decl. }}$ & $\begin{array}{c}\Delta \text { R.A. } \\
\left({ }^{\prime \prime}\right)\end{array}$ & $\begin{array}{c}\Delta \text { decl. } \\
\left({ }^{\prime \prime}\right)\end{array}$ \\
\hline $\mathrm{C}$ & $0.05 \pm 0.01$ & $-0.08 \pm 0.03$ & $-0.017 \pm 0.005$ & $-0.090 \pm 0.009$ \\
\hline IE & $0.07 \pm 0.05$ & $-0.22 \pm 0.06$ & $0.14 \pm 0.07$ & $-0.16 \pm 0.06$ \\
\hline IW & $-0.08 \pm 0.06$ & $0.02 \pm 0.05$ & $-0.10 \pm 0.07$ & $-0.02 \pm 0.04$ \\
\hline $\mathrm{OE}$ & $0.02 \pm 0.05$ & $-0.1 \pm 0.2$ & $0.22 \pm 0.06$ & $-0.1 \pm 0.1$ \\
\hline OW & $-0.36 \pm 0.05$ & $0.11 \pm 0.08$ & $-0.74 \pm 0.08$ & $0.1 \pm 0.1$ \\
\hline
\end{tabular}

Note.

${ }^{\mathrm{a}}$ The displacement of source $\mathrm{C}$ has been subtracted from the rest of the sources.

$1 \sigma$ uncertainties onto the R.A. and decl. axes. We perform this process on each of the $\mathrm{OE}$ and $\mathrm{OW}$ lobes at 5 and at $9 \mathrm{GHz}$ using $N=300$. Larger values of $N$ do not change the results appreciably.

In Figure 7, we summarize the PMs measured independently in the 9 and $5 \mathrm{GHz}$ images. Specifically, plotted are the velocities projected in the plane of the sky assuming a timescale of 6 years and a distance of $1.7 \mathrm{kpc}$. Red vectors indicate velocities derived from the displacement of the peak (Table 4) and black vectors those derived from maximizing cross-correlations (Table 5). The dashed line boxes indicate the regions used to calculate the cross-correlations. The bottom right inset of panel (b) in Figure 7 shows the PMs of the main OW lobe (calculated using cross-correlation maximization) and of the $\mathrm{W}_{1}$ knot (calculated as the difference between the positions given in Table 2). All PMs toward the OW lobe at 9 and $5 \mathrm{GHz}$ are consistent, except that the PM of the peak of the OW lobe at $9 \mathrm{GHz}$ is a factor of $\sim 2$ larger than the rest of the measurents toward OW.

We further note that the directions of the PMs of the OW and IW lobes lie along the approximate jet orientation pointing to the west. Figure 8 shows this more clearly by displaying all of the PM vectors of the western lobes (OW and IW) with the same origin. Figure 8 also shows the PMs of the eastern lobes (IE and OE). The direction of the eastern lobes' PMs have considerable more dispersion, although they also apparently scatter around the direction of the jet. One characteristic of the OE PMs which repeats at 5 and $9 \mathrm{GHz}$ is the widely different direction of the PM as determined from the peak compared to that determined from cross-correlations. The latter seems to have a strong component toward the north.

Another characteristic of the outer lobes, evident in Figures 5 and 6 , is that their morphology has changed somewhat between the 2008/09 and 2014/15 epochs. These changes can produce a PM signal not related to true displacements and they may account for the differences between the PMs determined using the two methods (peak position comparison and crosscorrelations maximization). As mentioned before, the value of $\rho_{\max }$ is a quantitative measure of the image similarity between the epochs. From Table 5, we note that in all cases $\rho_{\max }$ is close to 1 (columns 4 and 7), with the values at $5 \mathrm{GHz}$ being larger than those measured at $9 \mathrm{GHz}$. The latter is likely due to the angular resolution at $5 \mathrm{GHz}$ being lower that at $9 \mathrm{GHz}$, which helps to homogenize the morphology of the sources (at very low angular resolution, they would become two unresolved point sources). To determine how much does $\rho_{\max }$ decreases due to random noise, we calculate it between identical images of the OE and OW lobes but with the addition of simulated noise. We obtain values ranging between 0.98-0.99 and 0.94-0.98 for the simulated 5 and $9 \mathrm{GHz}$ data, respectively, showing that the measured values of $\boldsymbol{\rho}_{\max }$ are not fully explained by noise.

We conclude that while the high values of $\boldsymbol{\rho}_{\max }$ indicate that the bulk of the emission from the outer lobes has kept its morphology, there are still statistically significant changes in the shape between both epochs. Consequently, the differences in morphology between the two epochs for the outer lobes make it necessary to support their interpretation as PMs with additional evidence, for example, the bipolar anisotropy in the direction of the PMs shown in Figure 8.

Finally, Figure 9 shows images of the outer lobes at 17 and $19 \mathrm{GHz}$ (2014/15 epoch only). In part due to the likely filtering out of extended emission by the interferometer, the morphologies of lobes $\mathrm{OW}$ and $\mathrm{OE}$ are much more similar at these frequencies than they are at 9 or $5 \mathrm{GHz}$ : both outer lobes display an arcuate morphology, curved in all cases toward source C. Also plotted in Figure 9 are the peak position of the outer lobes determined from observations at the same frequency carried out in 2011 (Purser et al. 2016), confirming the PM of the OW lobe. The observed displacement of the OE lobe, on the other hand, seems similar to the PM determined from the cross-correlation method at $9 \mathrm{GHz}$, that is, rather perpendicular to the jet direction.

\section{DISCUSSION}

We adopt some of the nomenclature used by earlier studies and denote as G345.49+1.47 the HMYSO which dominates the luminosity of the massive clump identified with IRAS 16562-3959. Based on its luminosity, the HMSYO has $\approx 15$ $M_{\odot}$ while the mass of the IRAS 16562-3959 massive molecular clump is $\approx 1000 M_{\odot}$. In this work and in Guzmán et al. (2010), we identify five radio sources associated with G345.49+1.47: four lobes and source C. The position of the latter is coincident, within 0 !" 2 , with the position of the near-IR source identified in the $\mathrm{K}_{S}$ band in Section 3. It is also consistent, within 0."5, with the position of the HMYSO given 

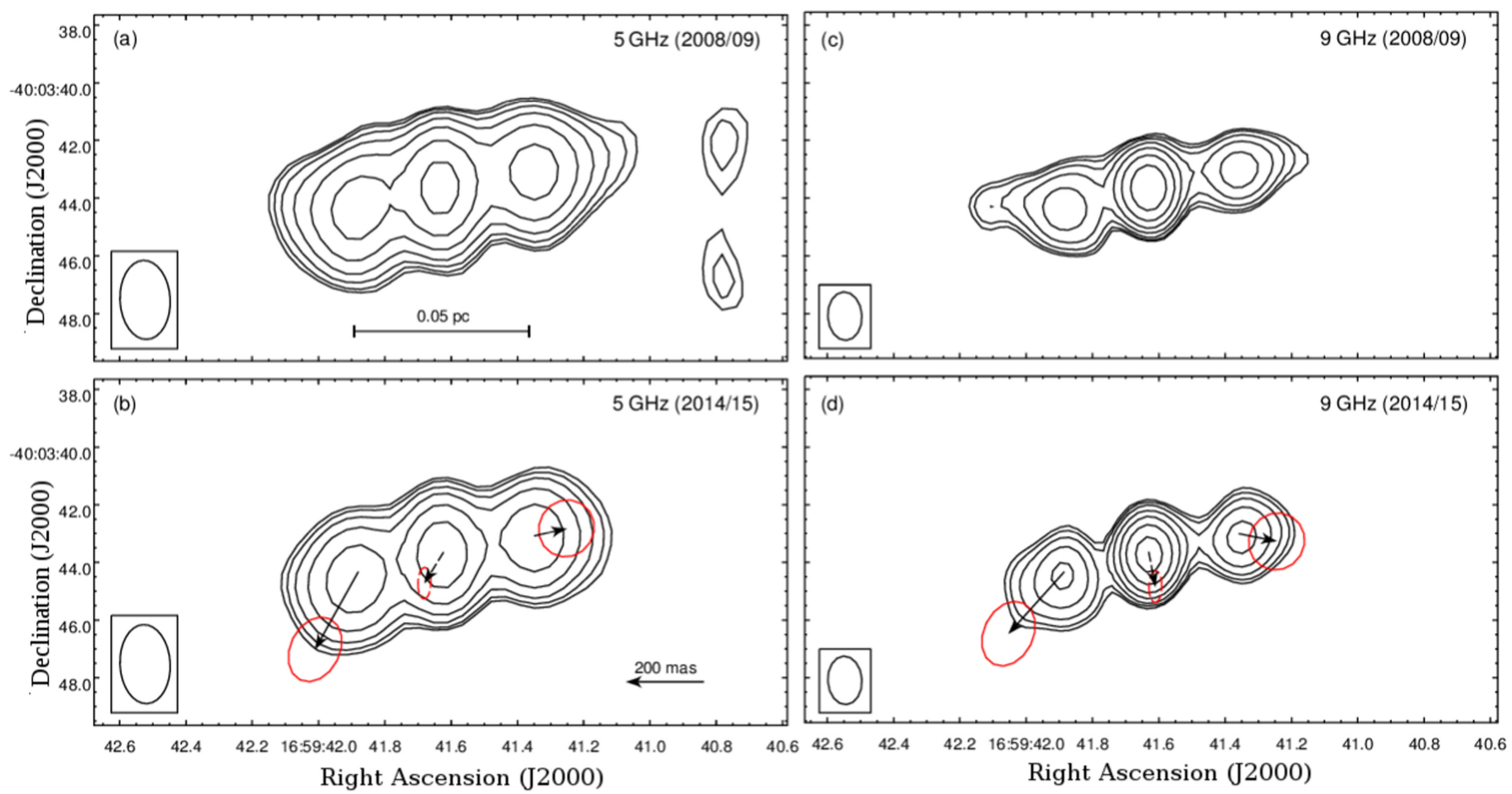

Figure 4. Source $\mathrm{C}$ and inner radio lobes around G345.49+1.47. Panels (a) and (b) show $5 \mathrm{GHz}$ contour maps from the 2008/09 and 2014/15 epochs, respectively. Contour levels: $\left(3+2^{i}\right) \times 50 \mu \mathrm{Jy}$ with $i=0-5$. Panels (c) and (d) show $9 \mathrm{GHz}$ contour maps from the 2008/09 and 2014/15 epochs, respectively. Contour levels: $\left(3+2^{i}\right) \times 50 \mu \mathrm{Jy}$ with $i=0-6$. Panels (b) and (d) show the PMs and their $1 \sigma$ uncertainty ellipses derived from the 5 and $9 \mathrm{GHz}$ data, respectively. PMs of source $\mathrm{C}$ - subtracted from the PMs of sources IE and IW-are marked using dashed lines. The velocity scale of the PM vectors is $100 \mathrm{~km} \mathrm{~s}^{-1}$ arcsec ${ }^{-1}$ (assuming a distance of $1.7 \mathrm{kpc}$ and a 6 years timescale).

by the rms Survey (Lumsden et al. 2013). Source C was also identified at $3 \mathrm{~mm}$ by Guzmán et al. (2014, their source 10).

In previous works (Guzmán et al. 2010, 2011, 2014), the radio emission between 1 and $100 \mathrm{GHz}$ arising from source $\mathrm{C}$ has been referred to as a "jet," nomenclature typically used to denote highly collimated streams of partially ionized, highvelocity $\left(>100 \mathrm{~km} \mathrm{~s}^{-1}\right)$ gas where the ionization is shock induced (see however, Tanaka et al. 2016). The HRL observations of Guzmán et al. (2014) indicate, however, that the radio emission from source $\mathrm{C}$ corresponds to a $\mathrm{HC} \mathrm{H}$ II region that may be expanding, but at a much lower velocity. The ionized gas is likely being excited by UV photons arising from G345.49+1.47. Thus, the "jet" nomenclature for source C is inappropriate.

\subsection{A Fast Protostellar Jet from an HC H II Region}

In star formation studies, mechanisms involving magnetic fields threading an accretion disk seem to better explain the acceleration and collimation of jets. Magneto-centrifugal acceleration sufficiently close to the star, for example, can produce velocities comparable to the escape speed. In addition, the magnetic field can collimate the ejected material close to the accreting star and explain the narrow cross-section of jets. Other explanations of jet formation face several theoretical and observational problems (Cabrit 2007, pp. 21-50).

1. Purely hydrodynamic mechanisms (e.g., Parker type winds, hydrodynamical nozzles) are able to accelerate the material only up to a few times the sound speed. Therefore, to explain velocities in excess of $100 \mathrm{~km} \mathrm{~s}^{-1}$, significant amounts of $\sim 10^{6} \mathrm{~K}$ gas are needed, which is not observed. On the other hand, acceleration by stellar radiation has proven insufficient to explain the momentum deposited in the associated molecular outflows.

2. In the low-mass case, collimation of the jet occurs relatively near the star, where the jet pressure is still too large to be confined by the ambient material. Recent observational evidence suggests that the jet collimation in high-mass stars also occurs near the HMYSO (Greenhill et al. 2013; Carrasco-González et al. 2015; Caratti o Garatti et al. 2016). It may be possible that the larger amount of material in high-mass star cores could help to confine the jet, but better angular resolution observations are still necessary to solve this issue.

The relevance of finding collimated jets toward HMYSOs is that they serve as signposts for disk accretion. Jets moving at velocities comparable to the escape velocity further indicate that the ejection mechanism is linked to accretion onto the central HMYSO, similar to the case for low-mass stars (Shu et al. 1987; Li et al. 2014, p. 173). PMs of radio lobes tracing jets have been measured in high-mass (Martí et al. 1998; Curiel et al. 2006; Rodríguez et al. 2008; Carrasco-González et al. 2010) and low-mass YSOs (Curiel et al. 1993; Rodríguez et al. 2000). In G345.49+1.47, the evidence for the radio lobes being excited by an underlying fast jet can be summarized as follows.

1. Most of the lobes display PMs. The magnitude of the PMs ranges between fast and highly significant signals for the OW lobe $\left(\gtrsim 500 \mathrm{~km} \mathrm{~s}^{-1}\right)$ to lower tangential 


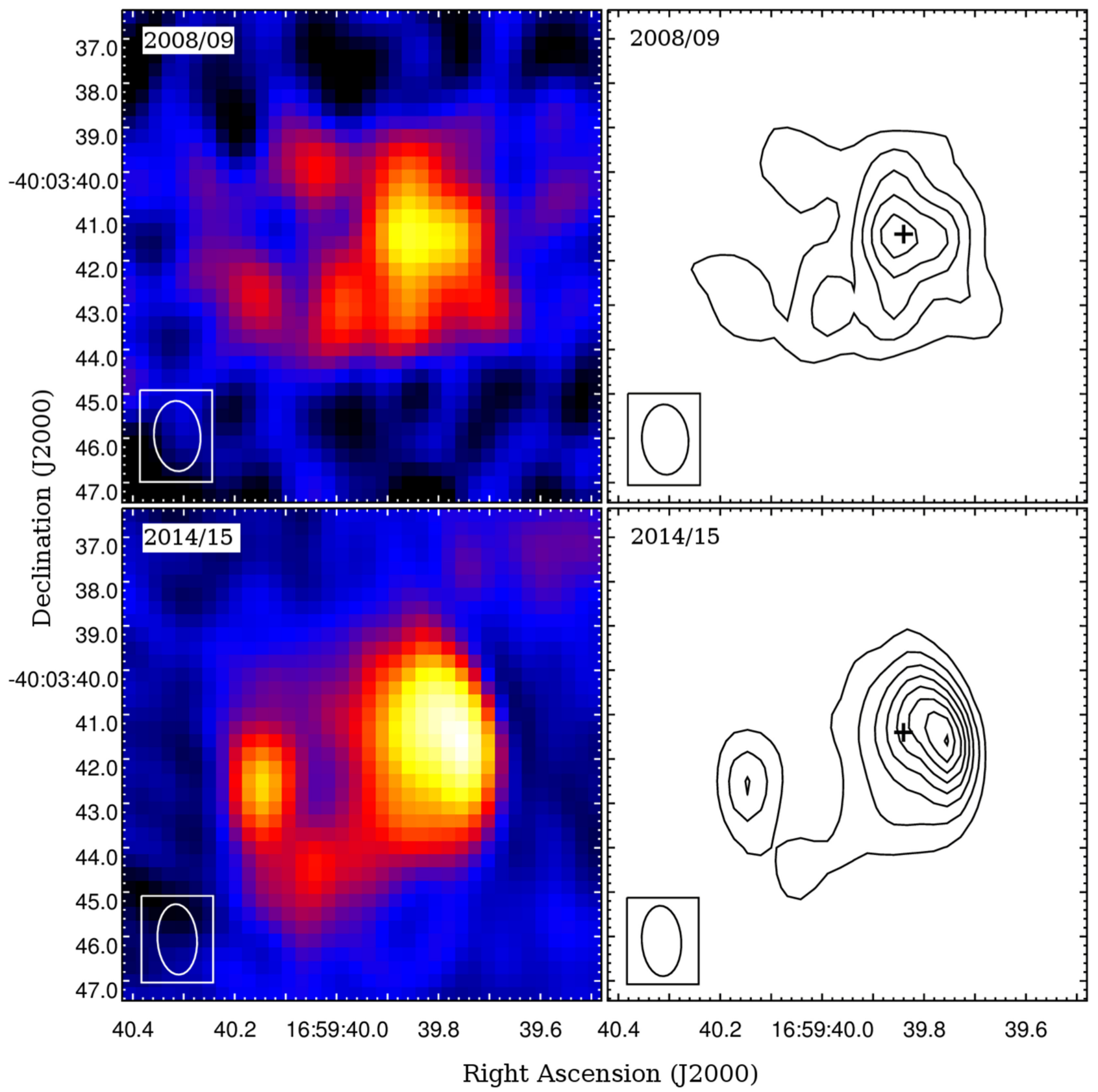

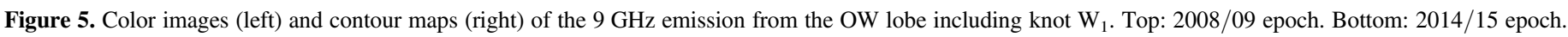

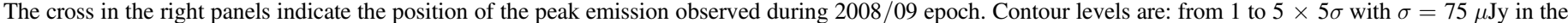
top right panel and from 1 to $8 \times 12 \sigma$ with $\sigma=17 \mu \mathrm{Jy}$ in the bottom right panel.

velocities (100-300 $\mathrm{km} \mathrm{s}^{-1}$ ) for the IE lobe and OE lobe at $9 \mathrm{GHz}$.

2. The lobes are aligned roughly in the east-west direction (P.A. $\approx 100^{\circ}$ ). As shown in Figures 7 and 8 , there is an evident anisotropy in the distribution of directions of the PMs: eastern lobes move roughly toward eastern directions (although with a large scatter) and western lobes move westerly. Both groups of lobes at each side of $\mathrm{G} 345.49+1.47$ recede from it, as expected in the case of a protostellar jet. This anisotropy is confirmed even by PMs detected with a low signal-to-noise ratio, such as those of the IW lobe.

3. The OW lobe displays an unmistakably bow-shock shape with an orientation consistent with moving away from
$\mathrm{G} 345.49+1.47$. The shape of the OE lobe at 9 and $5 \mathrm{GHz}$ is more complex, but its morphology at 17 and $19 \mathrm{GHz}$ suggests that it might also be a bow shock receding from $\mathrm{G} 345.49+1.47$.

4. A comparison of our $17 \mathrm{GHz}$ images with independent data taken during 2011 April by Purser et al. (2016) confirms the PM measurements reported in this work for the IE, IW, and OW lobes. In addition, evidence of fast shocks in the form of extended X-ray emission was found recently toward the OE lobe (V. A. Montes 2016, personal communication).

Based on the displacements on a timescale of 6 years observed at $9 \mathrm{GHz}$, we determine that the PMs of the OE, IE, IW, and OW lobes are $48 \pm 13,35 \pm 10,17 \pm 11$, and $64 \pm 12$ mas yr$^{-1}$, 


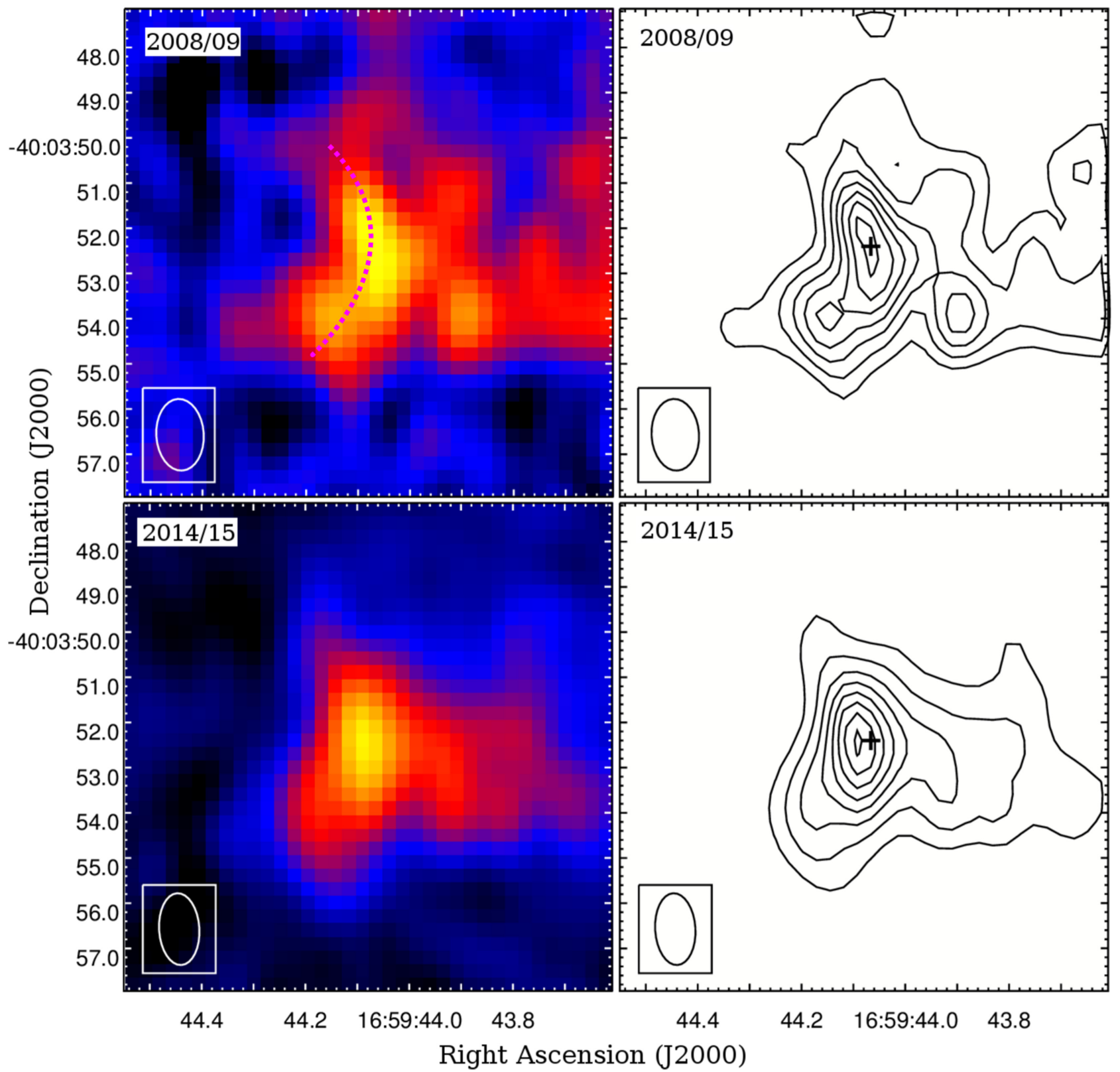

Figure 6. Color images (left) and contour maps (right) of the $9 \mathrm{GHz}$ emission from the OE lobe. Top: 2008/09 epoch. Bottom: 2014/15 epoch. The cross in the right panels show the position of the peak emission observed during 2008/09 epoch. The contour levels are: from 1 to $7 \times 2 \sigma$ with $\sigma=75 \mu \mathrm{Jy}$ in the top right panel and from 1 to $8 \times 10 \sigma$ with $\sigma=17 \mu \mathrm{Jy}$ in the bottom right panel. The dashed magenta curve in the top left panel indicates the arc-like shape of the emission discussed in Section 3.2.

Table 5

Displacements of Extended Lobes from Cross-correlation Maximization

\begin{tabular}{|c|c|c|c|c|c|c|}
\hline \multirow{2}{*}{ Source } & \multicolumn{3}{|c|}{$5 \mathrm{GHz}$} & \multicolumn{3}{|c|}{$9 \mathrm{GHz}$} \\
\hline & $\begin{array}{c}\Delta \text { R.A. } \\
\left({ }^{\prime \prime}\right)\end{array}$ & $\begin{array}{c}\Delta \text { decl. } \\
\left({ }^{\prime \prime}\right)\end{array}$ & $\rho_{\max }$ & $\begin{array}{c}\Delta \text { R.A. } \\
\left({ }^{\prime \prime}\right)\end{array}$ & $\begin{array}{c}\Delta \text { decl. } \\
\left({ }^{\prime \prime}\right)\end{array}$ & $\rho_{\max }$ \\
\hline OW & $-0.40 \pm 0.05$ & $0.10 \pm 0.08$ & 0.97 & $-0.38 \pm 0.07$ & $-0.06 \pm 0.08$ & 0.86 \\
\hline
\end{tabular}

respectively. For the OW lobe, we use the PM calculated by the cross-correlation method because it is in better agreement with that calculated at $5 \mathrm{GHz}$. Assuming a distance of $1.7 \mathrm{kpc}$, these PMs correspond to velocities of $390 \pm 100,280 \pm 80$,
$140 \pm 90$, and $520 \pm 100 \mathrm{~km} \mathrm{~s}^{-1}$ in the plane of the sky, respectively. The dynamical times of the lobes, calculated as the angular distance to source $\mathrm{C}$ divided by the PM, are $600 \pm 200$, $90 \pm 30,200 \pm 100$, and $330 \pm 60$ years for the OE, IE, IW, 

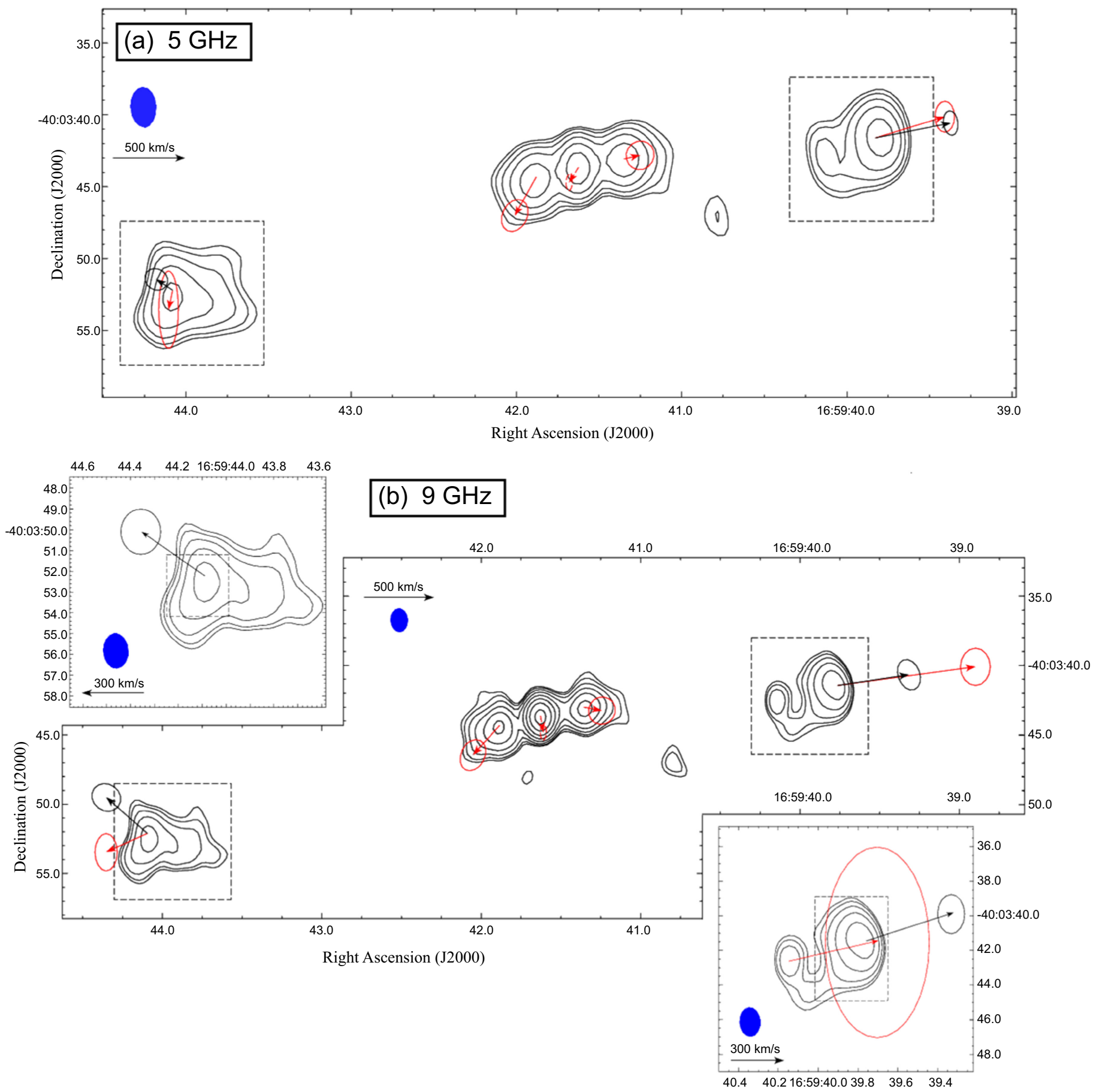

Figure 7. Contour maps and PMs of G345.49+1.47 at 5 and $9 \mathrm{GHz}$. Blue filled ellipses display the beam. Red vectors and ellipses indicate respectively the PMs and the uncertainty regions derived from displacements of the peak. The dashed line boxes and black vectors indicate the regions used for calculating the cross-correlation and the derived PMs, respectively. The velocity scale of the vectors is $1^{\prime \prime}$ per $100 \mathrm{~km} \mathrm{~s}^{-1}$. For sources IE, IW, and C, the PMs are the same as in Figure 4. PM of source $\mathrm{C}$ has been subtracted from that of the rest of the sources, at each frequency. Panel (a) $5 \mathrm{GHz}$ data. Contour levels: $2+2^{i} \times 50 \mu \mathrm{Jy}, i=1-6$. Panels (b) $9 \mathrm{GHz}$ data. Contour levels: $2+2^{i} \times 50 \mu \mathrm{Jy}, i=0-8$. Insets at the top left and bottom right corners of Panel (b) show zooms of the OE and OW lobes, respectively, and show the results of cross-correlation PMs over more restricted regions around the peak of each lobe. The bottom right inset shows the PM of $\mathrm{W}_{1}$.

and OW lobes, respectively. In principle, the underlying jet has been active (although likely with bursts of activity) during an interval spanning at least the range of dynamical times.

The relevance of G345.49+1.47 is that it shows that a collimated and fast jet can be generated from a HMYSO which is already producing ionizing radiation. In other cases of jets associated with HMYSOs with luminosities $>30,000 L_{\odot}$ like G343.1262-00.0620 (Rodríguez et al. 2008), IRAS 13481
-6124 (Kraus et al. 2010; Caratti o Garatti et al. 2015), or G35.2-0.7 N (Gibb et al. 2003), it is not clear that the HMYSO is already producing ionizing photons or that the central radio source corresponds to a photoionized region. Nevertheless, recent evidence presented by Beltrán et al. (2016) suggests that the central source of G35.2-0.7 $\mathrm{N}$ is a $\mathrm{HC} \mathrm{H}$ II region. Theoretical work describing disk accretion and jet ejection under the influence of ionizing radiation has been rather scarce. 


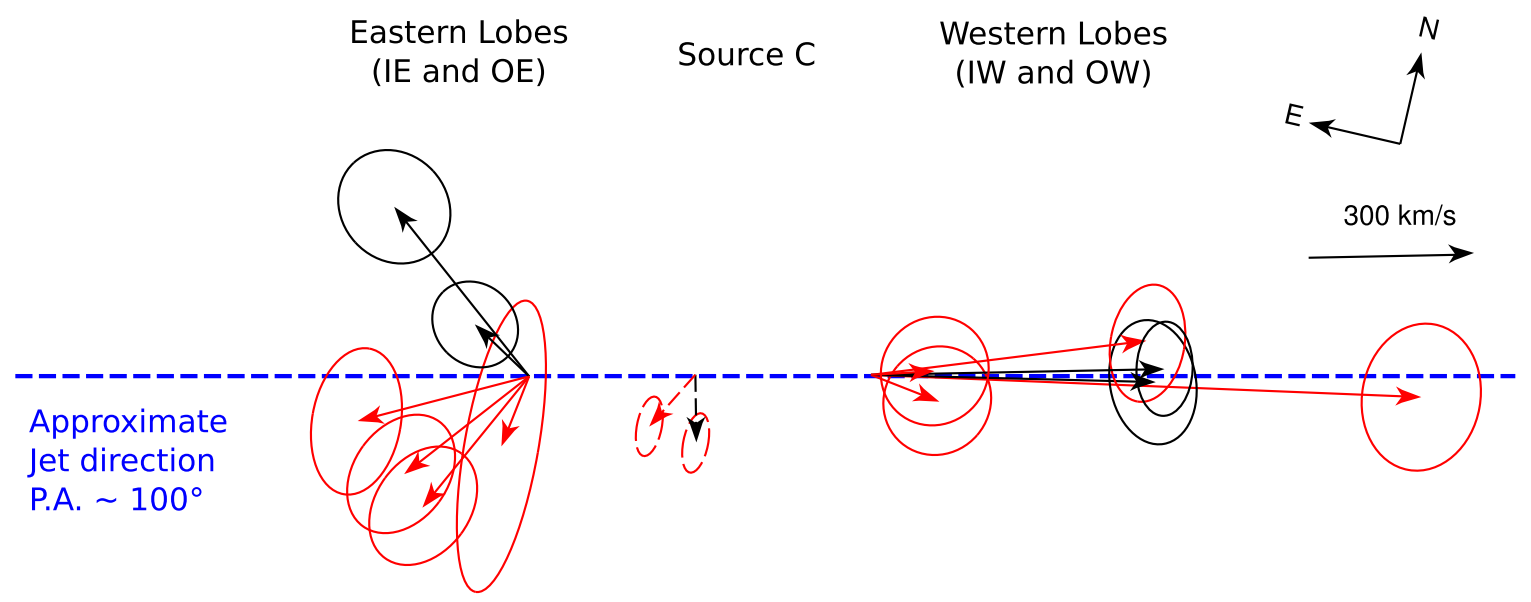

Figure 8. Diagram showing the direction and magnitude of the PMs. The colors of the vectors and ellipses follow the same convention as in Figure 7. The orientation of the diagram and the scale of the vectors and ellipses are given in the top right corner. The vectors are sorted into three groups: eastern lobes, Source C, and western lobes. In each of these groups, the PM vectors have the same origin.
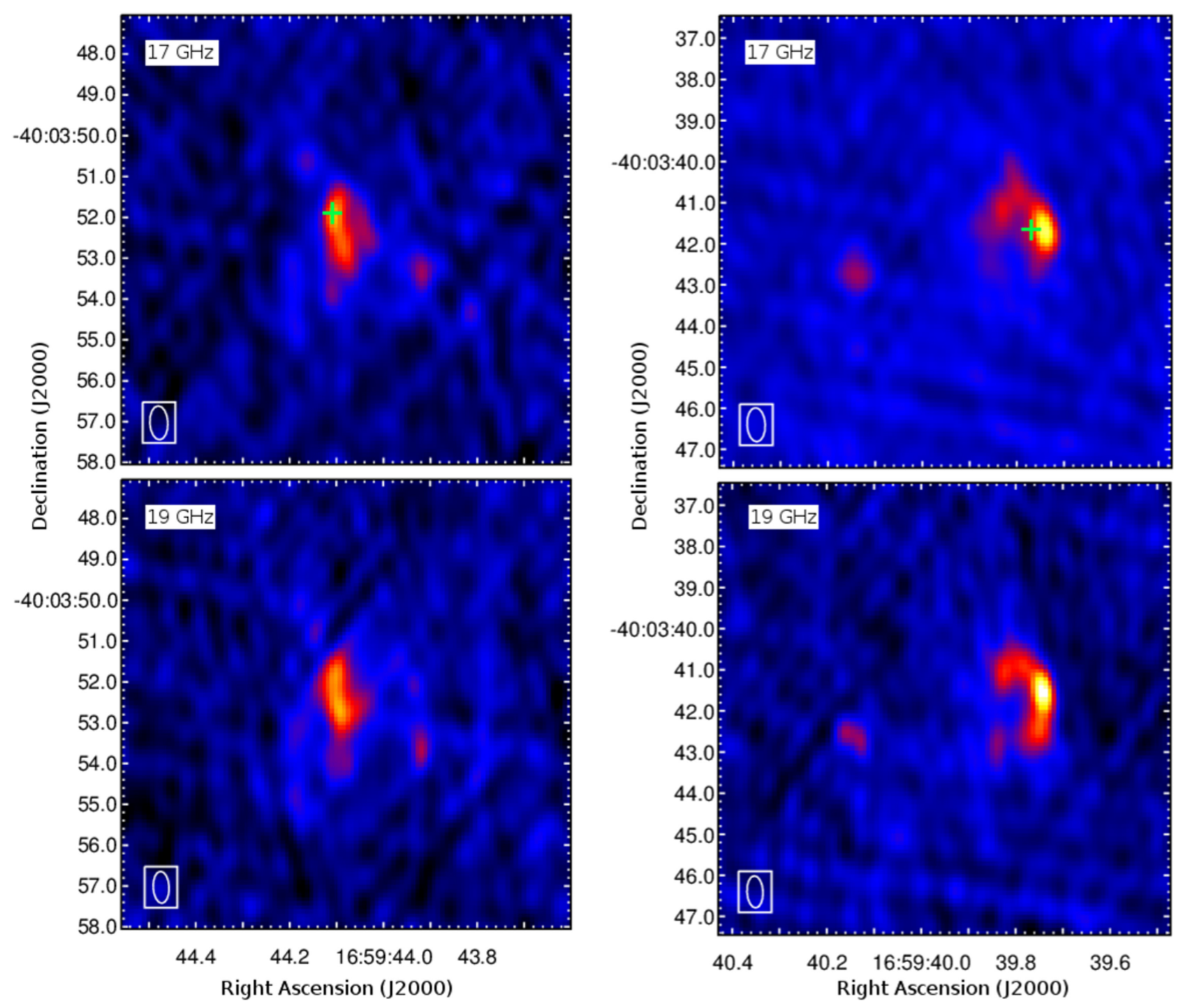

Figure 9. Radio continuum images of the outer lobes. Top panels: $17 \mathrm{GHz}$. Green crosses mark the 2011 peak position of OE and OW determined by Purser et al. (2016). Bottom panels: $19 \mathrm{GHz}$. Left panels: OE lobes, right panels: OW lobes. 
Tan \& McKee (2003) and Tanaka et al. (2016) investigated the ionization structure of a parameterized model of a collapsing massive core (including a disk and a disk wind) under UV photon injection. However, there are differences between our physical interpretation and theirs. In Tanaka et al. (2016), the ionized magnetically accelerated disk wind is the $\mathrm{HC} \mathrm{H}$ II region, predicting HRLs which are wider than the ones observed toward G345.49+1.47. In Guzmán et al. (2014), on the other hand, we suggest that the expansion of the $\mathrm{HC} \mathrm{H}$ II region is hydrodynamical (instead of magneto-centrifugal). We modeled the observed HRL widths with a combination of pressure and opacity broadening, and no contribution from bulk motions. The radio continuum and HRL emission of the jet itself is, in our interpretation, not detected.

\subsection{Nature of the Radio Emission}

\subsubsection{Source $C$ and the Lobes}

Based on the observations presented in this work, we provide further support to the simple model of source $\mathrm{C}$, as consisting of a biconical HC H II region (Guzmán et al. 2014). The flux density spectrum of source C (Figure 3, top panel) follows well the power-law fit in Guzmán et al. (2014), except perhaps by a slight decrement of the observed 17 and $19 \mathrm{GHz}$ fluxes with respect to the fit. This decrement is unlikely due to a hypothetical optically thin turn-off since, if such were the case, the HRL fluxes of source $\mathrm{C}$ would be inconsistent with the continuum flux measured at $3 \mathrm{~mm}$ (Guzmán et al. 2014). It is also apparent from Figure 3 that the spectral index of the fluxes at 17 and $19 \mathrm{GHz}$ is similar to the overall power-law fit. Hence, we attribute the offset either to a calibration error that produces an underestimation of $\sim 12 \%$ in the flux of source $\mathrm{C}$, or to intrinsic flux variations at 17 and $19 \mathrm{GHz}$ (Galván-Madrid et al. 2011).

In the lower panel of Figure 3, we plot the observed deconvolved size of source $\mathrm{C}$ as a function of frequency, showing a clear decrease with frequency. A power-law fit to the data gives a spectral index of $-1.1_{-0.4}^{+0.5}$. The apparent size of a biconical $\mathrm{HC} \mathrm{H}$ II region at a given frequency depends on the distance from the young star at which the free-free emission becomes optically thin. Using the notation of Reynolds (1986), this distance depends on frequency as $\nu^{2.1 / q_{\tau}}$. Considering that the aperture of the wind cavity is proportional to $r^{\epsilon}$, where $r$ is the distance to the central young $\operatorname{star}(\epsilon=1$ being a conical wind), $q_{\tau}=-3 \epsilon$ in the case of isothermal and non-accelerating winds. Therefore, the angular size of the source follows the relation $\theta \propto \nu^{-0.7 / \epsilon}$. We derive $\epsilon=0.6_{-0.1}^{+0.6}$ which is compatible with the range of parameters given by Guzmán et al. (Guzmán et al. 2014, Equation (16)). It is also compatible, within the uncertainty, with a conical wind.

The spectral energy distribution of the lobes seems to trace optically thin free-free emission. Power-law least squares fits to the flux densities of the east and west inner lobes indicate spectral indices of $-0.17 \pm 0.08$ and $-0.21 \pm 0.08$, respectively. The best-fit spectral indexes for the east and west outer lobes are $-0.22 \pm 0.03$ and $-0.32 \pm 0.03$, respectively. In each case the error bars correspond to $1 \sigma$ uncertainty derived from the method described in Lampton et al. (1976), and are based on flux uncertainties that do not include calibration errors. We note that the values of the spectral indices are not far from -0.1 , which is the value expected for optically thin thermal free-free emission. Furthermore, spectral indices between 5 and $9 \mathrm{GHz}$ are even flatter (closer to -0.1 ) compared with those calculated including the 17 and $19 \mathrm{GHz}$ fluxes, suggesting that a fraction of the flux at the high frequencies is being resolved out by the interferometer. We conclude that the emission from the lobes does not have spectral indices significatively lower than -0.1 , as would be expected in the case of optically thin synchrotron radiation. Further interferometer observations with a more complete uvplane coverage will allow us to better recover the extended emission associated with the lobes and to determine whether or not some fraction of it can be attributed to non-thermal processes.

\subsubsection{Sources 1, 2, and 3}

We detect source 1 at 9,17 , and $19 \mathrm{GHz}$. Because of the absence of IR or $3 \mathrm{~mm}$ counterparts, it is possible that its nature is extragalactic, possibly a $\mathrm{GHz}$ peaked radio-galaxy, as observed in other regions of star formation (Rodríguez et al. 2014; Dzib et al. 2015). According to the model of extragalactic radio source counts of AMI Consortium et al. (2011, at $15.7 \mathrm{GHz}$ ), the probability of detecting one or more radio sources brighter than $200 \mu \mathrm{Jy}$ inside the primary beam of the ATCA at $17 \mathrm{GHz}\left(140^{\prime \prime} \mathrm{FWHM}\right)$ is $\approx 0.2$.

Source 3 exhibits a peak flux density at $17 \mathrm{GHz}$ of $1.2 \mathrm{mJy}$. Since the probability of finding one or more radio sources brighter than $1 \mathrm{mJy}$ inside the $17 \mathrm{GHz}$ primary beam is $\lesssim 0.05$, it is unlikely that source 3 has an extragalactic origin. A more likely possibility is that source 3 corresponds to free-free emission from a low-mass YSO (see, for example, AMI Consortium et al. 2012), presumably forming part of the young embedded cluster associated with IRAS 16562-3959. This free-free emission is characterized by a rising spectrum until the turnover frequency, which in this case might be close to $20 \mathrm{GHz}$. If this were the case, then we would expect a $0.85 \mathrm{mJy}$ source at $3 \mathrm{~mm}$. Unfortunately, at $3 \mathrm{~mm}$ source 3 apparently blends with the $\sim 2$ mJy source 9 from Guzmán et al. (2014) located 1".2 (less than one beam size) to the east.

Finally, source 2 is associated with the IR object GLIMPSE $\mathrm{G} 345.4977+01.4668$ (Benjamin et al. 2003), the $3 \mathrm{~mm}$ source 18 in Guzmán et al. (2014), and the $6 \mathrm{GHz}$ methanol maser MMB345.498+1.467 (Caswell et al. 2010). Our observations provide new support for this source being a $\mathrm{HC} \mathrm{H}$ II region associated with a young high-mass star $\left(L_{\mathrm{bol}} \sim 10^{4} L_{\odot}\right)$. Keeping recombination equilibrium of this $\mathrm{HC} \mathrm{H}$ II region requires an ionizing photon flux $\geqslant 10^{45} \mathrm{~s}^{-1}$, consistent with a B2 star (Thompson 1984). The spectral index measured between 17 and $99 \mathrm{GHz}$ is 1.0 , similar to that observed at $3 \mathrm{~mm}$ (Guzmán et al. 2014).

\subsection{Jet and Shock Parameters}

The five radio sources $\mathrm{OE}, \mathrm{IE}, \mathrm{IW}, \mathrm{W}_{1}$, and $\mathrm{OW}$ are thought to trace shocks associated with the protostellar jet from G345.49+1.47. However, it is not clear whether these shocks are generated within the jet, or in the interaction zones with the ambient clump or with previously ejected material filling the outflow cavities. Theoretical models show that the density inside outflow cavities could be between 100 and 1000 times lower than the average density of the clump (e.g., Zhang et al. 2013).

We model the jet as having a circular cross-section of area $A_{\mathrm{j}}=\pi R_{\mathrm{j}}^{2}$, density $\rho_{\mathrm{j}}$, and velocity $V_{\mathrm{j}}$. This highly supersonic 
$\left(\mathcal{M}_{\mathrm{j}} \sim 100\right)$ jet is ramming through an stationary ambient medium of density $\rho_{\mathrm{a}}$ and driving a detached bow shock traveling through the ambient medium at velocity $V_{\mathrm{s}}$. The shock speed of the detached shock is not necessarily $V_{\mathrm{s}}$ except at its apex-because a large fraction of the shock front corresponds to weaker, oblique shocks. As a first approximation, we assume that the bow shock sweeps a transverse area $A_{\mathrm{s}}$. Conservation of momentum implies that (Chernin et al. 1994)

$$
\left(V_{\mathrm{j}}-V_{\mathrm{s}}\right)^{2} \rho_{\mathrm{j}} A_{\mathrm{j}}=V_{\mathrm{s}}^{2} \rho_{\mathrm{a}} A_{\mathrm{s}} .
$$

On the other hand, the escape velocity from a $15 M_{\odot}$ star-the estimated mass of the HMYSO — of radius $5.2 R_{\odot}$ (Davies et al. 2011; Mottram et al. 2011) is $V_{\mathrm{esc}} \approx 1050 \mathrm{~km} \mathrm{~s}^{-1}$. Under a wide range jet acceleration mechanisms, we expect the velocity of the jet not exceeding (at least by a large factor) the escape velocity of the accreting compact object (Ferreira et al. 2006). In fact, most jets appearing in several astrophysical situations have velocities comparable with $V_{\text {esc }}$ (Livio 2009, pp. 3-9). In the case of $\mathrm{G} 345.49+1.47, V_{\text {esc }}$ is within a factor of $\sim 2$ compared with the projected velocities measured toward the OE and OW lobes, 390 and $520 \mathrm{~km} \mathrm{~s}^{-1}$, respectively. Hereafter, we use the average and the unbiased standard deviation between these two values, $460 \pm 90 \mathrm{~km} \mathrm{~s}^{-1}$, as the projected shock velocity and its uncertainty, respectively. We can express this projected shock velocity as $V_{\mathrm{s}} \sin (i)$, where $i$ is the inclination angle between the jet direction and the line of sight. In Guzmán et al. (2011), we estimated $i=80^{\circ}$ from the molecular outflow. However, we prefer to leave $i$ as a free parameter because this estimation was obtained using relatively low angular resolution data and an extremely simple geometrical model of the outflow. Note also that $i$ may vary along the jet due to precession, which may help to explain the difference of transverse velocities between the inner and the outer lobes. We summarize the conditions expected between the shock, jet, and escape velocities by

$$
460 \pm 90 \mathrm{~km} \mathrm{~s}^{-1} / \sin (i) \approx V_{\mathrm{s}} \leqslant V_{\mathrm{j}} \lesssim V_{\mathrm{esc}} \approx 1050 \mathrm{~km} \mathrm{~s}^{-1} .
$$

These conditions imply that $i \geqslant 26^{\circ}$ and, in combination with Equation (3),

$$
\frac{\rho_{\mathrm{a}}}{\rho_{\mathrm{j}}}=\alpha\left(\frac{V_{\mathrm{j}}}{V_{\mathrm{s}}}-1\right)^{2} \leqslant(1.6 \pm 0.7) \alpha,
$$

where $\alpha=A_{\mathrm{j}} / A_{\mathrm{s}}$. The mass and momentum rates produced by one side of the jet, on the other hand, are given by

$$
\begin{gathered}
\dot{M}_{\mathrm{j}}=\pi R_{\mathrm{j}}^{2} \rho_{\mathrm{j}} V_{\mathrm{j}}, \\
\dot{P}_{\mathrm{j}}=\pi R_{\mathrm{j}}^{2} \rho_{\mathrm{j}} V_{\mathrm{j}}^{2}=\dot{M}_{\mathrm{j}} V_{\mathrm{j}},
\end{gathered}
$$

respectively.

We define $\epsilon$ as the fraction of the jet's momentum being shed to the ambient medium through the shock. We approximate this fraction using (Chernin et al. 1994)

$$
\epsilon=\frac{\rho_{\mathrm{a}} V_{\mathrm{s}}^{2} A_{\mathrm{s}}}{\rho_{\mathrm{j}} V_{\mathrm{j}}^{2} A_{\mathrm{j}}}=\frac{1}{\alpha}\left(1-\frac{V_{\mathrm{s}}}{V_{\mathrm{j}}}\right)^{2},
$$

where the second equality is derived from Equation (3). We can constrain the rate of momentum being injected in the ambient medium $\left(\epsilon \dot{P}_{\mathrm{j}}\right)$ using the radio emission of the lobes $\left(F_{\nu}\right)$ under the assumption that it corresponds to shocked ambient material using

$$
\begin{aligned}
\left(\frac{F_{\nu} d^{2}}{\mathrm{Jy} \mathrm{kpc}^{2}}\right)= & 2.5 \epsilon \dot{P}_{\mathrm{j}}\left(\frac{\nu}{\mathrm{GHz}}\right)^{-0.1}\left(\frac{V_{\mathrm{s}}}{500 \mathrm{~km} \mathrm{~s}^{-1}}\right)^{-0.32} \\
& \times\left(\frac{T_{e}}{10^{4} \mathrm{~K}}\right)^{0.45} M_{\odot} \mathrm{km} \mathrm{s}^{-1} \mathrm{yr}^{-1} .
\end{aligned}
$$

This equation assumes that the radio emission corresponds to optically thin and completely ionized free-free gas (see Curiel et al. 1989 and Cabrit \& Bertout 1992). For the OW bow shock (the $\mathrm{OE}$ shock is similar), $F_{9 \mathrm{GHz}}=5.7 \pm 0.6 \mathrm{mJy}$. We assume that the uncertainty is dominated by absolute calibration uncertainties, which we conservatively assume to be $10 \%$ (the measurement uncertainty is much less). Assuming $d=1.7 \mathrm{kpc}$ and $T_{e}=7000 \mathrm{~K}$ (Guzmán et al. 2014), and using $V_{\mathrm{s}}=460 \pm$ $90 / \sin (i) \mathrm{km} \mathrm{s}^{-1}$, we conclude that $\epsilon \dot{P}_{\mathrm{j}}=(9 \pm 1) \times 10^{-3}$ $(\sin (i))^{-0.32} M_{\odot} \mathrm{km} \mathrm{s}^{-1} \mathrm{yr}^{-1}$, where the uncertainty is derived from the the mean shock velocity and the radio flux at $9 \mathrm{GHz}$.

Furthermore, we estimate the jet's dynamical time, $t_{\mathrm{dyn}, \mathrm{j}}$, as the projected mean distance of the outer lobes from the HMYSO $(0.21 \pm 0.05 \mathrm{pc})$ divided by the projected mean velocity of the outer lobes, obtaining $t_{\mathrm{dyn}, \mathrm{j}} \approx 450 \pm 140$ years. The inclination is not relevant for this calculation. We then estimate how much momentum the OW bow shock has injected in the ambient medium as $t_{\mathrm{dyn}, \mathrm{j}} \times \epsilon \dot{P}_{\mathrm{j}}$, obtaining $4 \pm 1(\sin (i))^{-0.32} M_{\odot} \mathrm{km} \mathrm{s}^{-1}$. The momentum of the molecular outflow associated with $\mathrm{G} 345.49+1.47$, on the other hand, is $2 P_{\mathrm{CO}} \approx 15 \pm 2$ $M_{\odot} \mathrm{km} \mathrm{s}^{-1}$ (Guzmán et al. 2011), where the factor of two takes into account that we are summing the blue and redshifted sides. We assume that the uncertainty of the outflow momentum is dominated by a $10 \%$ uncertainty in the absolute calibration, but we stress that there are other sources of bias need to be kept in mind (Cabrit \& Bertout 1990; Downes \& Cabrit 2007). We follow the prescriptions given by Downes \& Cabrit (2007) and drop the usual $\cos (i)^{-1}$ inclination correction factor. This factor strongly overestimates the real momentum of jet-driven molecular outflows because an important fraction of the gas moves in transverse directions with respect to the jet.

Because the momentum injected by the OW bow shock is comparable within a factor of 2 with that of one side of the molecular outflow, we conclude that at most a few bow shocks similar to the OW lobe could have driven the entire molecular flow reported in Guzmán et al. (2011). In particular, since the sizes of the jet and of the molecular outflow are similar, it is possible that the latter was driven by the OE and OW shocks. We note, however, that the dynamical time of the molecular outflow (calculated according to the "perpendicular" timescale defined in Downes \& Cabrit 2007) is 2000 years, larger than that of the outer lobes. In addition, the well developed bipolar near-IR cavities and the $\mathrm{K}_{S}$ emission extending much farther than the molecular flows (Guzmán et al. 2011, Figure 5) seem to indicate that the ejection of material is older than $t_{\mathrm{dyn}, \mathrm{j}}$. Further observations will determine whether or not the observed extended $\mathrm{K}_{S}$ emission corresponds to shocked $\mathrm{H}_{2}$ evidencing older outflow activity.

The density of the pre-shocked material can be estimated from the peak brightness of the lobes as follows. Using the averaged 
$17-19 \mathrm{GHz}$ data, we find that the peak intensity of the OW lobe is $6.8 \pm 0.7 \times 10^{-4} \mathrm{Jy} \mathrm{beam}^{-1}$ at a mean frequency of $18 \mathrm{GHz}$. This intensity is equivalent to a brightness temperature of $7.7 \pm 0.8 \mathrm{~K}$, using a beam solid angle of $0.38 \operatorname{arcsec}^{2}$. At $9 \mathrm{GHz}$, on the other hand, the measured peak brightness temperature of the OW lobe is $17 \pm 2 \mathrm{~K}$ with a beam of $1.61 \operatorname{arcsec}^{2}$. We can estimate the intrinsic peak brightness temperature $\left(T_{0}\right)$ of the OW lobe using the relation $T_{\mathrm{a}}=T_{0} /\left(1+\Omega_{b} / \Omega_{\mathrm{s}}\right)$ (Wilson et al. 2013, chapter 7), where $\Omega_{\mathrm{s}}$ is the solid angle subtended by the source, $\Omega_{b}$ is the beam size, and $T_{\mathrm{a}}$ is the measured brightness temperature. Assuming that the emission comes from optically thin free-free gas, the quotient between the measured peak temperatures at frequencies $\nu_{1}$ and $\nu_{2}$ is given by $\left(\nu_{1} / \nu_{2}\right)^{-2.1}\left(1+\Omega_{b, 2} / \Omega_{\mathrm{s}}\right) /\left(1+\Omega_{b, 1} / \Omega_{\mathrm{s}}\right)$. From this relation and the peak temperature quotient between 9 and $18 \mathrm{GHz}$, we derive $\Omega_{\mathrm{s}} \sim 1.0 \pm 0.3 \operatorname{arcsec}^{2}$. Hence, $T_{0} \approx 11 \pm 3 \mathrm{~K}$. Based on the free-free emission model from shocks of Curiel et al. (1989), the brightness temperature at $18 \mathrm{GHz}$ is given in the optically thin limit by

$$
T_{\mathrm{b}}=0.105 \mathrm{~K}\left(\frac{\rho_{\mathrm{a}}}{10^{3} \mu \mathrm{cm}^{-3}}\right)\left(\frac{V_{\mathrm{s}}}{100 \mathrm{~km} \mathrm{~s}^{-1}}\right)^{1.68}\left(\frac{T_{e}}{10^{4} \mathrm{~K}}\right)^{0.45},
$$

where $T_{e}$ is the temperature of the shock-ionized gas and $\rho_{\mathrm{a}}$ is the ambient density. Using $V_{\mathrm{s}}=460 \pm 90 / \sin (i) \mathrm{km} \mathrm{s}^{-1}$, $T_{\mathrm{b}}=T_{0}=11 \pm 3 \mathrm{~K}$, and assuming $T_{e}=7000 \mathrm{~K}$, we derive $\rho_{\mathrm{a}}=(9 \pm 4) \times 10^{3} \mu(\sin (i))^{1.68} \mathrm{~cm}^{-3}$. This is also comparable with the density derived from Ghavamian \& Hartigan (1998, Figure 7). Note that in this calculation, we are assuming that the shock velocity is given by the PM of the OW lobe. This approximation is valid because we are using the peak brightness temperature occurring at the apex of the bow shock where the shock front is close to normal.

Using this value of the pre-shocked material, we obtain the effective size of the OW shock, $R_{\mathrm{s}}$, defined as $\sqrt{A_{\mathrm{s}} / \pi}$. Clearly, $\alpha=R_{\mathrm{j}}^{2} / R_{\mathrm{s}}^{2}$. From Equations (7) and (8) we derive

$$
R_{\mathrm{s}}=\left(\frac{\epsilon \dot{P}_{\mathrm{j}}}{\pi \rho_{\mathrm{a}} V_{\mathrm{s}}^{2}}\right)^{1 / 2} \approx 1400 \pm 200 \mathrm{au}
$$

where using the values of $\epsilon \dot{P}_{\mathrm{j}}$ and $\rho_{\mathrm{a}}$ estimated above cancels the dependence on inclination and on $V_{\mathrm{s}}$. The value for $R_{\mathrm{s}}$ obtained in Equation (11) is within a $20 \%$ of the half angular size of the OW lobe, which is $\approx 1^{\prime \prime}$, equivalent to $1700 \mathrm{au}$. This consistency is not entirely trivial since Equation (11) was not derived using measured sizes from the images but using the PMs, the total flux of the lobe, the peak brightness temperature, and the quotient between the brightness temperatures at two frequencies. Hereafter, we assume that $R_{\mathrm{S}}$ ranges between 1400 and 1700 au. Using these values for $R_{\mathrm{s}}$, we can constrain $\alpha$ by assuming that the $\mathrm{W}_{1}$ lobe corresponds to an inner shock inside the jet. Since $\mathrm{W}_{1}$ is unresolved and our smallest synthesized beam has a FWHM with an equivalent physical size of $960 \mathrm{au}$, we assume that the transverse size of the jet $\left(2 R_{\mathrm{j}}\right)$ is smaller than half of the beam size, or $R_{\mathrm{j}} \leqslant 240 \mathrm{au}$.

We can derive a rough lower limit for $R_{\mathrm{j}}$ by noting that the spectrum of the emission from the $\mathrm{W}_{1}$ knot does not seem to arise from optically thick gas. While the flux density of $\mathrm{W}_{1}$ - fixed by the observations, see Table 3 -is $\propto n_{e}^{2} R_{\mathrm{j}}^{3}$, the optical depth of the knot is given by $\tau \propto n_{e}^{2} R_{\mathrm{j}}$. By requiring $\tau \leqslant 1$, we derive that
$R_{\mathrm{j}} \geqslant 50 \pm 10 \mathrm{au}$. Combining the previously derived constraints on $R_{\mathrm{s}}$ and $R_{\mathrm{j}}$, we finally derive $9 \times 10^{-4} \leqslant \alpha \leqslant 0.03$.

Finally, under the circumstances suggested in this section, the brightness of the bow shocks and its associated Mach disk should be similar (Hartigan 1989). We can estimate the distance between the Mach disk and the bow shock using the expression $\xi R_{\mathrm{j}} c_{\mathrm{s}}^{0} / 2 V_{\mathrm{j}}$ (Raga \& Cantó 1998), where $c_{\mathrm{s}}^{0} \sim 10 \mathrm{~km} \mathrm{~s}^{-1}$ is the sound speed of the ionized gas and $\xi$ is close to unity for $\rho_{\mathrm{j}} \approx \rho_{\mathrm{a}}$. Since $c_{\mathrm{s}}^{0} / V_{\mathrm{j}} \leqslant 0.02 \sin (i)$ for $\mathrm{G} 345.49+1.47$, we expect the Mach disk and bow-shock emission to be blended in our observations. Based on this, we rule out $\mathrm{W}_{1}$ as being the Mach disk associated with the OW lobe.

\subsection{Mass Accretion Rate}

We can obtain a lower bound on the mass outflow rate $\dot{M}_{\mathrm{j}}$ of the jet using Equations (4), (5), (6), (10), and (11):

$$
\begin{aligned}
\dot{M}_{\mathrm{j}} & =\pi R_{\mathrm{j}}^{2} \rho_{\mathrm{j}} V_{\mathrm{j}} \geqslant \frac{\pi R_{\mathrm{s}}^{2} \rho_{\mathrm{a}} V_{\mathrm{s}}}{1.6 \pm 0.7} \\
& =(1.2 \pm 0.8) \times 10^{-5} \sin (i)^{0.68} M_{\odot} \mathrm{yr}^{-1}
\end{aligned}
$$

To estimate the accretion rate onto the HMYSO, $\dot{M}_{\text {acc }}$, we can assume it is related with the jet's mass outflow rate through $2 \dot{M}_{\mathrm{j}}=f_{\mathrm{a}} \dot{M}_{\mathrm{acc}}$, where and $f_{\mathrm{a}}$ typically ranges between 0.1 and 0.4 (e.g., Tomisaka 1998; Banerjee \& Pudritz 2006; Seifried et al. 2012). Unfortunately, with the current data it does not seem possible to give an upper limit on $\dot{M}_{\mathrm{j}}$ because the density of the jet is not constrained by the observations. In principle, it could be possible that $\rho_{\mathrm{j}} \gg \rho_{\mathrm{a}}$ (with a large $\dot{M}_{\mathrm{j}}$ ) and $\epsilon \ll 1$, that is, a very dense jet piercing the clump depositing a very small fraction of its momentum in the medium.

We can constrain from below the accumulated mass in the central object using

$$
M_{\star}=2 \int_{0}^{t_{\star}} \frac{\dot{M}_{\mathrm{j}}}{f_{\mathrm{a}}} d t \geqslant \frac{12 \pm 8}{f_{\mathrm{a}}}\left(\frac{t_{\star}}{\mathrm{Myr}}\right) \sin (i)^{0.68} M_{\odot},
$$

where $t_{\star}$ is the HMYSO age. The last inequality in Equation (13) uses (12) and assumes that $\dot{M}_{\mathrm{j}}$ and $f_{\mathrm{a}}$ have been constant during the entire HMYSO lifetime. Since G345.49+1.47 is in the HC $\mathrm{H}$ II region phase, we assume that its age is $\approx 10^{5}$ years (Guzmán et al. 2012). Although with quite large uncertainties, we can conclude that the jet characteristics are roughly consistent with disk accretion rates of the order of a few times $10^{-4} M_{\odot} \mathrm{yr}^{-1}$ (assuming $f_{\mathrm{a}}=0.1-0.2$ ), which are sufficient to account for the current mass of the HMYSO.

\section{SUMMARY}

We have observed the HMYSO G345.49+1.47 in radio continuum at $5,9,17$, and $19 \mathrm{GHz}$ using the ATCA between 2014 October and 2015 May. Our main results are summarized as follows.

1. By comparing observations separated by 6 years, we determine that the PMs of the OE, IE, IW, and OW lobes are $48 \pm 13,35 \pm 10,17 \pm 11$, and $64 \pm 12 \mathrm{mas} \mathrm{yr}^{-1}$, respectively. Assuming a distance of $1.7 \mathrm{kpc}$, these PMs correspond to velocities of $390 \pm 100,280 \pm 80$, $140 \pm 90$, and $520 \pm 100 \mathrm{~km} \mathrm{~s}^{-1}$ in the plane of the sky, respectively. 
2. The PMs of the lobes are directed away from G345.49 +1.47 . In addition, the morphology of the outer lobesespecially of that of the western outer lobe-are consistent with detached bow shocks.

3. We interpret these results as evidence that the radio lobes are produced in working surfaces associated with a highly collimated protostellar jet. The radio lobes match the IR extended emission, which is likely tracing the illuminated inner outflow cavity containing the jet. The jet's velocity is comparable with the HMYSO escape velocity, which is also the case for jets detected toward low-mass YSOs. We propose that a mechanism of jet ejection and disk accretion similar to that of low-mass stars is acting in G345.49+1.47. The presence of ionizing radiation or the $\mathrm{HC} \mathrm{H}$ II region does not hinder this process.

4. We observe emission arising from a previously undetected ionized knot $\left(\mathrm{W}_{1}\right)$ in the jet path located between the outer and inner west lobes.

5. We determined that the size spectral index of the $\mathrm{HC} \mathrm{H}$ II region (source $\mathrm{C}$ ) is $-1.1_{-0.4}^{+0.5}$, which is compatible with a bipolar (and possibly biconical) ionized region.

6 . The momentum injected by the bow shocks in the past $\sim 500$ years is comparable to the observed momentum of the molecular outflow. We propose that the previously reported bipolar molecular outflow reflects only a relatively recent fraction of the history of ejection from G345.49+1.47 (Guzmán et al. 2011). Further observations will determine whether or not there are indications of older ejection of material located farther from the HMYSO.

7. The characteristics of the jet are roughly consistent with disk accretion rates of the order of $10^{-4} M_{\odot} \mathrm{yr}^{-1}$, which are sufficient to account for the current estimated mass of G345.49+1.47 in $\sim 10^{5}$ years.

A.E.G. acknowledges support from FONDECYT through grant 3150570. G.G. acknowledges support from CONICYT through project PFB-06. The authors thank an anonymous referee for the constructive comments and suggestions which helped to improve this manuscript.

\section{APPENDIX \\ ANGULAR RESOLUTION MATCHING}

In order to compare the features in maps made with different beam shapes, it is convenient to transform the images to a common beam. This is important for disentangling real morphological differences from artifacts introduced by the instrument and the observing technique. When both images are characterized by Gaussian and radially symmetric beams, the beam sizes are matched by convolving the image associated with the smaller beam, characterized by a FWHM $=\theta$, with a symmetric Gaussian of FWHM $=\delta$, such that $\theta^{2}+\delta^{2}=\Theta^{2}$, where $\Theta$ is the FWHM of the larger Gaussian beam. The larger Gaussian beam is the optimal common beam shape which can be attained without deconvolving the images.

Finding an optimal convolving beam when the two images are characterized by elliptical Gaussian beams is more involved. This is usually the case in interferometry, where the images' resolution are determined by their synthesized beams. In order to reach a common resolution, one possibility is to convolve the images with each other's beams. However, this solution is not optimal and it does not match with the procedure described above for circular beams.

In this appendix, we derive a solution which is optimal in the sense it minimizes the sum in quadrature of the major and minor semiaxes of the convolving beams. Denoting by $\alpha$, $B_{\text {maj }}$, and $B_{\text {min }}$ the P.A., major, and minor FWHM axes, respectively, of an elliptical Gaussian beam, we define $\boldsymbol{M}_{1}:=R\left(\alpha_{1}\right)\left(\begin{array}{ll}B_{1, \text { maj }}^{2} & 0 \\ 0 & B_{1, \text { min }}^{2}\end{array}\right) R\left(-\alpha_{1}\right)$, where $R\left(\alpha_{1}\right)$ is the rotation matrix associated with $\alpha_{1}$. Subscripts 1 and 2 refer to quantities for each one of the images. Let $\boldsymbol{D}=\boldsymbol{M}_{1}-\boldsymbol{M}_{2}$ and $d_{1}$ and $d_{2}$ be its eigenvalues. We define $\boldsymbol{B}_{1}=$ $R\left(\alpha_{D}\right) \Lambda_{1} R\left(-\alpha_{D}\right)$, where $R\left(\alpha_{D}\right)$ is the rotation matrix associated with the normalized eigenvectors of $\boldsymbol{D}$ and $\Lambda_{1}$ is a diagonal matrix defined as $\left(\Lambda_{1}\right)_{i i}=-\min \left(d_{i}, 0\right)$ for $i=1,2$. We define $\boldsymbol{B}_{2}$ in the same way, but with diagonal terms equal to $\max \left(d_{i}, 0\right)$. Matrices $\boldsymbol{B}_{1}$ and $\boldsymbol{B}_{2}$ define the Gaussians that match the beams of both images via convolution with $\boldsymbol{M}_{1}$ and $\boldsymbol{M}_{2}$, respectively (that is, $\boldsymbol{B}_{1}+\boldsymbol{M}_{1}=\boldsymbol{B}_{2}+\boldsymbol{M}_{2}$ ). We still need to prove that they are the optimal solutions.

Clearly, $\boldsymbol{B}_{1}$ and $\boldsymbol{B}_{2}$ are positive semi-definite (PSD) matrices and $\boldsymbol{B}_{2}-\boldsymbol{B}_{1}=\boldsymbol{D}$. Every PSD matrix represents a (maybe degenerate) Gaussian beam. We now prove that among all other PSD matrices $X$ such that $X+\boldsymbol{D}$ is PSD, $\boldsymbol{B}_{1}$ minimizes the sum in quadrature of its eigenvalues. Let $r_{\mathrm{j}}$ be the eigenvectors associated with the negative (or negatives) eigenvalues of $\boldsymbol{D}$ and $F(X)$ the sum of the squares of the eigenvalues of a matrix $X$. Then $F(X) \geqslant \sum_{j}\left(r_{\mathrm{j}}^{T} X r_{\mathrm{j}}\right)^{2}$. Since $\boldsymbol{D}+X$ is PSD, we have that $d_{\mathrm{j}}+r_{\mathrm{j}}^{T} X r_{\mathrm{j}} \geqslant 0$, and because $d_{\mathrm{j}}<0 \leqslant r_{\mathrm{j}}^{T} X r_{\mathrm{j}}$, we conclude that $\left(r_{\mathrm{j}}^{T} X r_{\mathrm{j}}\right)^{2} \geqslant d_{\mathrm{j}}^{2}$. Therefore $F(X) \geqslant \sum_{\mathrm{j}} d_{\mathrm{j}}^{2}=F\left(\boldsymbol{B}_{1}\right)$. Similarly, for each PSD matrix $Y$ such that $Y-D$ is PSD, $\mathrm{B}_{2}$ minimizes $F$. Therefore, if $X$ and $Y$ are PSD and fulfill $\boldsymbol{M}_{1}+X=\boldsymbol{M}_{2}+Y$, then $F(X)+F(Y) \geqslant$ $F\left(\boldsymbol{B}_{1}\right)+F\left(\boldsymbol{B}_{2}\right)$. Therefore, $\boldsymbol{B}_{1}$ and $\boldsymbol{B}_{2}$ minimize the sum in quadrature of their eigenvalues and fulfill $\boldsymbol{B}_{1}+\boldsymbol{M}_{1}=\boldsymbol{B}_{2}+\boldsymbol{M}_{2}$ (see also Horn \& Johnson 2012, Section 5.2).

Matrices $\boldsymbol{B}_{1}$ and $\boldsymbol{B}_{2}$ define the convolving beams. The P.A. is $\alpha_{D}$ and the FWHM axes squared are given in the diagonal terms of $\Lambda_{i}$. If $\boldsymbol{B}_{1}$ (respectively, $\boldsymbol{B}_{2}$ ) is 0, then we only need to convolve the second (first) image by the beam described by the parameters of $\boldsymbol{B}_{2}\left(\boldsymbol{B}_{1}\right)$. If one of the convolving beam's axes is zero (as it may happen when both beams are of similar size but have different orientations), then the convolving beam is degenerate and infinitely narrow in one direction. In practice, we limit the minimum axis of the convolving beam to 3 pixels.

\section{REFERENCES}

AMI Consortium, Ainsworth, R. E., Scaife, A. M. M., et al. 2012, MNRAS, 423, 1089

AMI Consortium, Davies, M. L., Franzen, T. M. O., et al. 2011, MNRAS, 415, 2708

Bally, J., \& Zinnecker, H. 2005, AJ, 129, 2281

Banerjee, R., \& Pudritz, R. E. 2006, ApJ, 641, 949

Beltrán, M. T., Cesaroni, R., Moscadelli, L., et al. 2016, arXiv:1606.03943

Benjamin, R. A., Churchwell, E., Babler, B. L., et al. 2003, PASP, 115, 953

Cabrit, S. 2007, in Jets from Young Stars I: Models and Constraints, Vol. 723 ed. J. Ferreira, C. Dougados, \& E. Whelan (Berlin: Springer)

Cabrit, S., \& Bertout, C. 1990, ApJ, 348, 530

Cabrit, S., \& Bertout, C. 1992, A\&A, 261, 274

Caratti o Garatti, A., Stecklum, B., Linz, H., Garcia Lopez, R., \& Sanna, A. 2015, A\&A, 573, A82

Caratti o Garatti, A., Stecklum, B., Weigelt, G., et al. 2016, A\&A, 589, L4 
Carrasco-González, C., Rodríguez, L. F., Torrelles, J. M., Anglada, G., \& González-Martín, O. 2010, AJ, 139, 2433

Carrasco-González, C., Torrelles, J. M., Cantó, J., et al. 2015, Sci, 348, 114 Caswell, J. L., Fuller, G. A., Green, J. A., et al. 2010, MNRAS, 404, 1029

Chernin, L., Masson, C., Gouveia dal Pino, E. M., \& Benz, W. 1994, ApJ, 426, 204

Condon, J. J. 1997, PASP, 109, 166

Condon, J. J., Cotton, W. D., Greisen, E. W., et al. 1998, AJ, 115, 1693

Curiel, S., Ho, P. T. P., Patel, N. A., et al. 2006, ApJ, 638, 878

Curiel, S., Rodriguez, L. F., Bohigas, J., et al. 1989, ApL\&C, 27, 299

Curiel, S., Rodriguez, L. F., Moran, J. M., \& Canto, J. 1993, ApJ, 415, 191

Davies, B., Hoare, M. G., Lumsden, S. L., et al. 2011, MNRAS, 416, 972

De Pree, C. G., Peters, T., Mac Low, M.-M., et al. 2014, ApJL, 781, L36

Downes, T. P., \& Cabrit, S. 2007, A\&A, 471, 873

Dzib, S. A., Loinard, L., Rodríguez, L. F., et al. 2015, ApJ, 801, 91

Ferreira, J., Dougados, C., \& Cabrit, S. 2006, A\&A, 453, 785

Galván-Madrid, R., Peters, T., Keto, E. R., et al. 2011, MNRAS, 416, 1033

Galván-Madrid, R., Rodríguez, L. F., Ho, P. T. P., \& Keto, E. 2008, ApJL, 674, L33

Garay, G., Brooks, K. J., Mardones, D., \& Norris, R. P. 2003, ApJ, 587, 739

Garay, G., \& Lizano, S. 1999, PASP, 111, 1049

Ghavamian, P., \& Hartigan, P. 1998, ApJ, 501, 687

Gibb, A. G., Hoare, M. G., Little, L. T., \& Wright, M. C. H. 2003, MNRAS, 339, 1011

Greenhill, L. J., Goddi, C., Chandler, C. J., Matthews, L. D., \& Humphreys, E. M. L. 2013, ApJL, 770, L32

Guzmán, A. E., Garay, G., \& Brooks, K. J. 2010, ApJ, 725, 734

Guzmán, A. E., Garay, G., Brooks, K. J., Rathborne, J., \& Güsten, R. 2011, ApJ, 736, 150

Guzmán, A. E., Garay, G., Brooks, K. J., \& Voronkov, M. A. 2012, ApJ, 753, 51

Guzmán, A. E., Garay, G., Rodríguez, L. F., et al. 2014, ApJ, 796, 117

Hartigan, P. 1989, ApJ, 339, 987

Hennebelle, P., Commerçon, B., Joos, M., et al. 2011, A\&A, 528, A72

Hoare, M. G., Kurtz, S. E., Lizano, S., Keto, E., \& Hofner, P. 2007, in Protostars and Planets V, ed. B. Reipurth, D. Jewitt, \& K. Keil (Tucson, AZ: Univ. of Arizona Press), 181

Horn, R., \& Johnson, C. 2012, Matrix Analysis (Cambridge: Cambridge Univ. Press)

Hosokawa, T., \& Omukai, K. 2009, ApJ, 691, 823

Johnston, K. G., Robitaille, T. P., Beuther, H., et al. 2015, ApJL, 813, L19

Keto, E. 2007, ApJ, 666, 976

Keto, E., \& Wood, K. 2006, ApJ, 637, 850

Klaassen, P. D., Testi, L., \& Beuther, H. 2012, A\&A, 538, A140

Kraus, S., Hofmann, K.-H., Menten, K. M., et al. 2010, Natur, 466, 339

Kuiper, R., Klahr, H., Beuther, H., \& Henning, T. 2011, ApJ, 732, 20

Kurtz, S. E. 2000, RMxAA, 9, 169

Lampton, M., Margon, B., \& Bowyer, S. 1976, ApJ, 208, 177

Larson, R. B., \& Starrfield, S. 1971, A\&A, 13, 190

Li, Z.-Y., Banerjee, R., Pudritz, R. E., et al. 2014, in Protostars and Planets VI, ed. H. Beuther, R. S. Klessen, C. P. Dullemond, \& T. Henning (Tucson, AZ: Univ. of Arizona Press), 173

Livio, M. 2009, in Protostellar Jets in Context, ed. K. Tsinganos, T. Ray, \& M. Stute (Berlin: Springer)
López, C., Bronfman, L., May, J., Nyman, L.-A., \& Garay, G. 2011, A\&A, 534, A131

Lumsden, S. L., Hoare, M. G., Urquhart, J. S., et al. 2013, ApJS, 208, 11

Martí, J., Rodríguez, L. F., \& Reipurth, B. 1998, ApJ, 502, 337

Masqué, J. M., Rodríguez, L. F., Araudo, A., et al. 2015, ApJ, 814, 44

McMullin, J. P., Waters, B., Schiebel, D., Young, W., \& Golap, K. 2007, in ASP Conf. Ser. 376, Astronomical Data Analysis Software and Systems XVI, ed. R. A. Shaw, F. Hill, \& D. J. Bell (San Francisco, CA: ASP), 127

Minniti, D., Lucas, P. W., Emerson, J. P., et al. 2010, NewA, 15, 433

Mottram, J. C., Hoare, M. G., Davies, B., et al. 2011, ApJL, 730, L33

Mottram, J. C., Hoare, M. G., Lumsden, S. L., et al. 2007, A\&A, 476, 1019

Nakano, T. 1989, ApJ, 345, 464

Norberg, P., \& Maeder, A. 2000, A\&A, 359, 1025

Peters, T., Klaassen, P. D., Mac Low, M.-M., Klessen, R. S., \& Banerjee, R. 2012, ApJ, 760, 91

Purser, S. J. D., Lumsden, S. L., Hoare, M. G., et al. 2016, MNRAS, 460, 1039

Raga, A. C., \& Cantó, J. 1998, in Astrophysical Jets: Open Problems, ed. S. Massaglia \& G. Bodo (Amsterdam: Gordon and Breach Science Publishers), 201

Raga, A. C., Noriega-Crespo, A., Rodríguez-González, A., et al. 2012, ApJ, 748, 103

Reipurth, B., Raga, A. C., \& Heathcote, S. 1996, A\&A, 311, 989

Reynolds, S. P. 1986, ApJ, 304, 713

Rodríguez, L. F., Carrasco-González, C., Montes, G., \& Tapia, M. 2014, AJ, 148,20

Rodríguez, L. F., Delgado-Arellano, V. G., Gómez, Y., et al. 2000, AJ, 119,882

Rodríguez, L. F., Moran, J. M., Franco-Hernández, R., et al. 2008, AJ, 135,2370

Sánchez-Monge, Á, Cesaroni, R., Beltrán, M. T., et al. 2013, A\&A, 552, L10

Sault, R. J., Teuben, P. J., \& Wright, M. C. H. 1995, in ASP Conf. Ser. 77, Astronomical Data Analysis Software and Systems IV, ed. R. A. Shaw, H. E. Payne, \& J. J. E. Hayes (San Francisco, CA: ASP), 433

Schwartz, R. D. 1978, ApJ, 223, 884

Seifried, D., Pudritz, R. E., Banerjee, R., Duffin, D., \& Klessen, R. S. 2012, MNRAS, 422, 347

Shu, F. H., Adams, F. C., \& Lizano, S. 1987, ARA\&A, 25, 23

Tan, J. C., Beltrán, M. T., Caselli, P., et al. 2014, in Protostars and Planets VI, ed. H. Beuther, R. S. Klessen, C. P. Dullemond, \& T. Henning (Univ. of Arizona Press), 149

Tan, J. C., \& McKee, C. F. 2003, Star Formation at High Angular Resolution, ed. M. Burton, R. Jayawardhana, \& T. Bourke, arXiv:astro-ph/0309139

Tanaka, K. E. I., Tan, J. C., \& Zhang, Y. 2016, ApJ, 818, 52

Thompson, R. I. 1984, ApJ, 283, 165

Tomisaka, K. 1998, ApJL, 502, L163

Wilson, T., Rohlfs, K., \& Huettemeister, S. 2013, Tools of Radio Astronomy (Berlin, Heidelberg: Springer)

Wilson, W. E., Ferris, R. H., Axtens, P., et al. 2011, MNRAS, 416, 832

Wolfire, M. G., \& Cassinelli, J. P. 1987, ApJ, 319, 850

Zapata, L. A., Tang, Y.-W., \& Leurini, S. 2010, ApJ, 725, 1091

Zhang, Y., Tan, J. C., \& Hosokawa, T. 2014, ApJ, 788, 166

Zhang, Y., Tan, J. C., \& McKee, C. F. 2013, ApJ, 766, 86

Zinnecker, H., \& Yorke, H. W. 2007, ARA\&A, 45, 48 